\title{
Blocking fatty acid-fueled mROS production within macrophages alleviates acute gouty inflammation
}

\author{
Christopher J. Hall, ${ }^{1}$ Leslie E. Sanderson, ${ }^{1}$ Lisa M. Lawrence, ${ }^{1}$ Bregina Pool, ${ }^{2}$ Maarten van der Kroef, ${ }^{1}$ Elina Ashimbayeva, ${ }^{1}$ \\ Denver Britto, ${ }^{1}$ Jacquie L. Harper, ${ }^{3}$ Graham J. Lieschke, ${ }^{4}$ Jonathan W. Astin, ${ }^{1}$ Kathryn E. Crosier, ${ }^{1}$ \\ Nicola Dalbeth, ${ }^{2}$ and Philip S. Crosier ${ }^{1}$ \\ 'Department of Molecular Medicine and Pathology and ${ }^{2}$ Department of Medicine, Faculty of Medical and Health Sciences, University of Auckland, Auckland, New Zealand. ${ }^{3}$ Malaghan Institute for Medical \\ Research, Wellington, New Zealand. ${ }^{4}$ Australian Regenerative Medicine Institute, Monash University, Victoria, Australia.
}

\begin{abstract}
Gout is the most common inflammatory arthritis affecting men. Acute gouty inflammation is triggered by monosodium urate (MSU) crystal deposition in and around joints that activates macrophages into a proinflammatory state, resulting in neutrophil recruitment. A complete understanding of how MSU crystals activate macrophages in vivo has been difficult because of limitations of live imaging this process in traditional animal models. By live imaging the macrophage and neutrophil response to MSU crystals within an intact host (larval zebrafish), we reveal that macrophage activation requires mitochondrial ROS (mROS) generated through fatty acid oxidation. This mitochondrial source of ROS contributes to NF-KB-driven production of IL-1 $\beta$ and TNF- $\alpha$, which promote neutrophil recruitment. We demonstrate the therapeutic utility of this discovery by showing that this mechanism is conserved in human macrophages and, via pharmacologic blockade, that it contributes to neutrophil recruitment in a mouse model of acute gouty inflammation. To our knowledge, this study is the first to uncover an immunometabolic mechanism of macrophage activation that operates during acute gouty inflammation. Targeting this pathway holds promise in the management of gout and, potentially, other macrophage-driven diseases.
\end{abstract}

\section{Introduction}

Gout, the most common inflammatory arthritis in men (1), is a prototypic acute sterile inflammatory disease. Hyperuricemia leading to monosodium urate (MSU) crystal deposition triggers recurrent attacks of acute joint inflammation (flares) (2). Flares are a key concern to patients, as they cause extreme joint pain and difficulty with daily activities including walking, sleeping, and working (3). Current antiinflammatory medications used to treat and prevent flares (NSAIDs, colchicine, and corticosteroids) are limited by potential drug toxicities and gaps in therapeutic efficacy (4-6). Furthermore, these agents are only modestly effective; for example, when NSAIDs are used to treat acute gout, only approximately $50 \%$ of patients experience a major clinical response within 2 days (7). A thorough understanding of how MSU crystals trigger inflammation is necessary to uncover new pathways to pharmacologically target and prevent extremely painful and disabling gout flares (8).

A gout flare is initiated and driven by the cellular arm of the innate immune system, in particular macrophages and neutrophils (9). MSU crystals stimulate tissue-resident macrophages to produce proinflammatory cytokines including IL-1 $\beta$ (through activation of the NLRP3 inflammasome) (10) and TNF- $\alpha$ and IL-6, which contribute to neutrophil recruitment, a hallmark of acute gouty inflammation (9). Not all individuals with hyperuricemia develop gout, and, in patients with gout, flares are intermittent despite

Conflict of interest: The authors have declared that no conflict of interests exists. Submitted: April 14, 2017; Accepted: February 7, 2018.

Reference information: / Clin Invest. 2018;128(5):1752-1771.

https://doi.org/10.1172/JCI94584. persistent articular MSU crystal deposition, suggesting that other factors may synergize with MSU crystals to trigger inflammation (4). One of these factors is fatty acids (FAs), which are a metabolic consequence of excessive alcohol and food consumption (risk factors for gout flares). Flares of acute joint inflammation in patients with gout correlate with elevated serum FA levels $(11,12)$. In addition, FAs have been shown to augment MSU crystal-stimulated IL-1 $\beta$ secretion from human peripheral blood mononuclear cells (PBMCs) and murine macrophages (13). This work connects FAs with macrophage-driven acute gouty inflammation and highlights the need to examine MSU crystal-stimulated macrophages in the context of an intact microenvironment, where synergistic factors, like FAs, are preserved.

Macrophages display remarkable plasticity with respect to their metabolic and immunological phenotypes (14). Macrophage functional plasticity is, at least in part, governed by changes in cellular metabolism and alternate use of metabolic fuels, including FAs (14). Mitochondria-derived ROS (mROS) are important by-products of cellular metabolism that can couple adaptive changes in core metabolic processes with inflammatory pathways (15). Whether FAs help fuel the activation of macrophages in response to MSU crystals to trigger gouty inflammation is unknown. Exploiting the live imaging potential of larval zebrafish, we have previously shown that macrophages exploit FA oxidation (FAO) to fuel bactericidal mROS production in a process requiring a mitochondrial enzyme encoded by immunoresponsive gene 1 (irg1) (16). Irg1 encodes cis-aconitic acid decarboxylase (CAD), an enzyme that catalyzes production of the metabolite itaconic acid from the TCA cycle intermediate cis-aconitate (17). In a murine air 
A

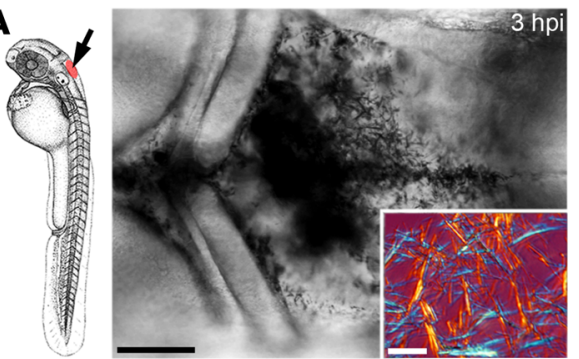

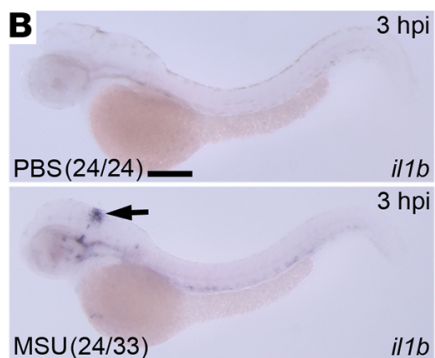

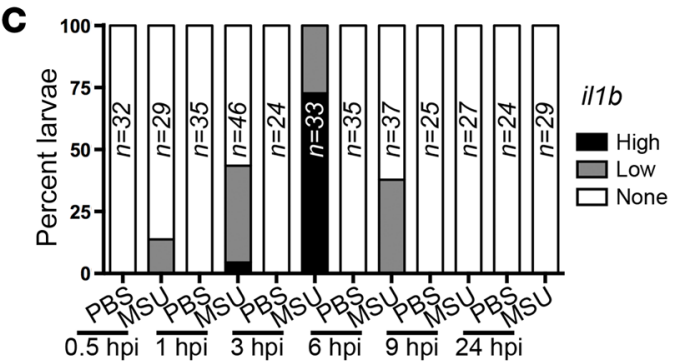

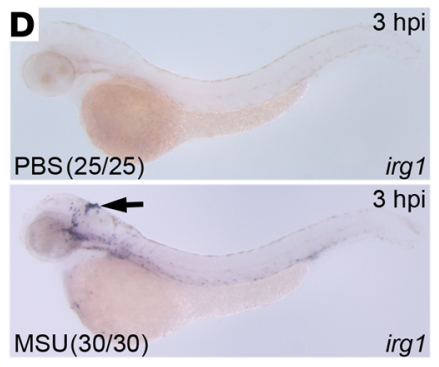
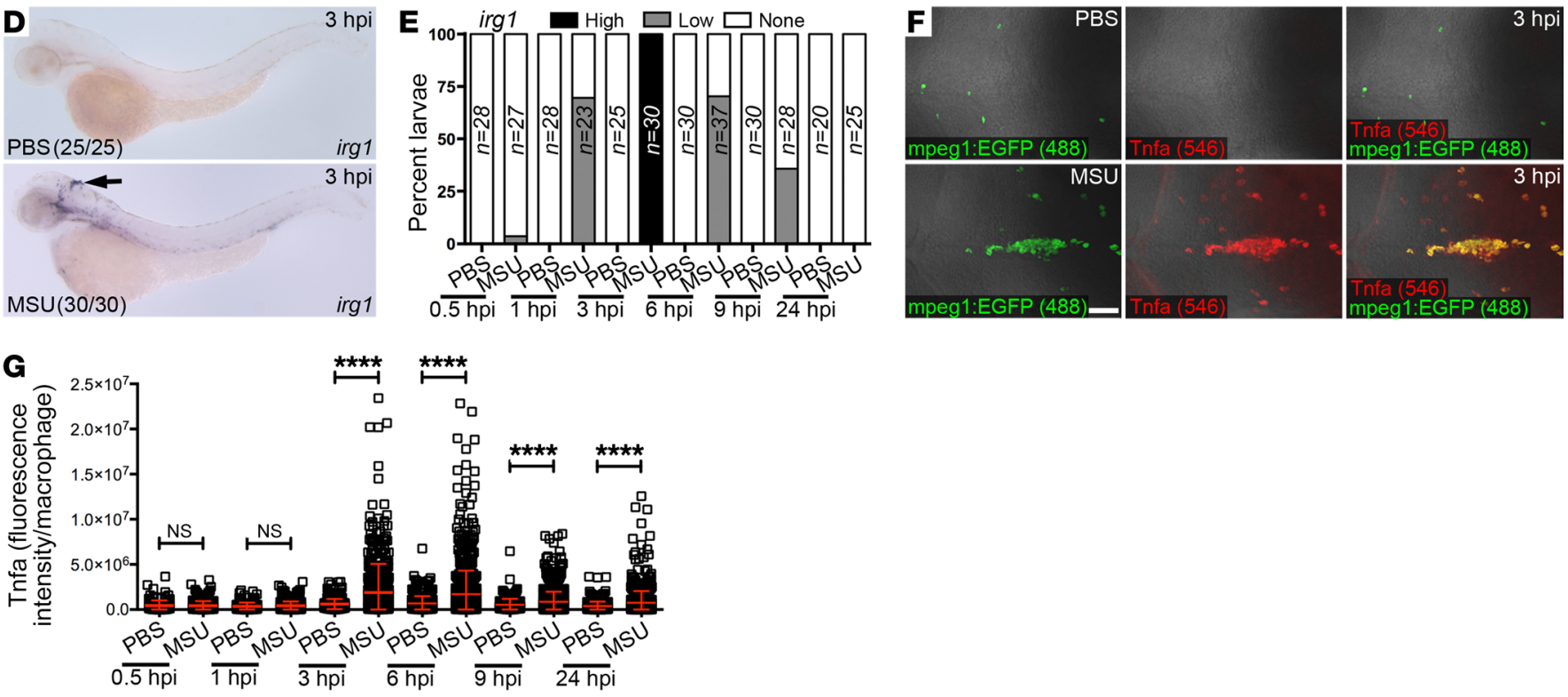

Figure 1. MSU crystals activate zebrafish macrophages. (A) Injection site and dorsal hindbrain view following MSU crystal injection (inset shows crystals under polarized light). (B) Expression of il1b within PBS- and MSU crystal-injected larvae. (C) Temporal quantification of illb expression was categorized as high (B), low (Supplemental Figure 1C), or no expression (None). (D) Expression of irg1 within PBS- and MSU crystal-injected larvae. (E) Temporal quantification of irg1 expression: high (D), low (Supplemental Figure 1C), or no expression. (F) Immunofluorescence of Tnfa within the hindbrain of PBS- and MSU crystal-injected 1\% DMSO-treated Tg(mpeg1:EGFP) larvae and temporal quantification in individual macrophages (C). $n=15$ larvae/treatment. Arrows mark illb/irg1 expression in the hindbrain. The numbers in parentheses in $\mathbf{B}$ and $\mathbf{D}$ indicate the frequency of larvae with the indicated phenotype. Data were pooled from 2 independent experiments and represent the mean \pm SD. ${ }^{* * * *} P<0.0001$, by Student's $t$ test. Scale bars: $50 \mu \mathrm{m}$ (A and $\mathbf{F}), 5 \mu \mathrm{m}$ (A, inset), and $100 \mu \mathrm{m}$ (B). Magnification value $\times 6$ (D).

pouch model of acute gouty inflammation, Irg1 was identified as one of the most highly overexpressed genes within macrophages (18). To date, a role for Irg1 during MSU crystal-driven macrophage activation has not been described.

Here, we developed a larval zebrafish model of acute gouty inflammation to explore the macrophage and neutrophil response to MSU crystals within an intact host. This live imaging approach revealed that macrophages utilize FAO to fuel the elevated production of mROS. This MSU crystal-stimulated, FAO-fueled mROS production was dependent on JAK2/ STAT3-driven irg1 expression, promoted macrophage-specific Il1b and Tnfa production (the zebrafish orthologs of IL-1 $\beta$ and TNF- $\alpha$ ) through the NF- $\mathrm{KB}$ signaling pathway, and was necessary for neutrophil recruitment. We also provide evidence supporting the conservation of this immunometabolic mechanism of macrophage activation in human THP-1 monocytes/macrophages. Here, we demonstrate the utility of the larval zebrafish model as a tool to identify new therapeutics to treat and prevent acute gouty inflammation, and show that drugs that block this metabolic mechanism of MSU crystal-driven macrophage acti- vation in zebrafish have conserved activities in THP- 1 cells and inhibited neutrophil recruitment in an in vivo mouse model of acute gouty inflammation.

\section{Results}

The larval zebrafish innate immune cell response to MSU crystals is highly conserved with the response described in mammals, including sensitivity to conventional antiinflammatory treatments. We first investigated whether larval zebrafish macrophages and neutrophils were responsive to MSU crystals. Throughout this study, MSU crystals were microinjected into the hindbrain ventricle of day-2 post fertilization (dpf) larvae, a well-established injection site that facilitates live imaging of innate immune cells at the single-cell level (16) and provides inflammatory foci for the examination of neutrophil recruitment. Passing MSU crystals through finegauge needles followed by sonication resulted in crystal lengths of $2.32 \pm 1.53 \mu \mathrm{m}$ (mean $\pm \mathrm{SD}, n=85$ ) that readily dispersed throughout the hindbrain ventricle following microinjection (Figure 1A and Supplemental Figure 1A; supplemental material available online with this article; https://doi.org/10.1172/JCI94584DS1). 

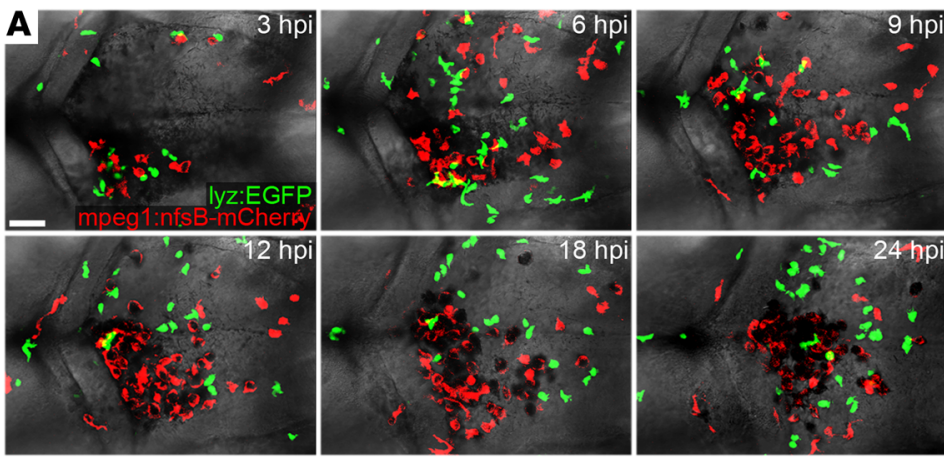

B

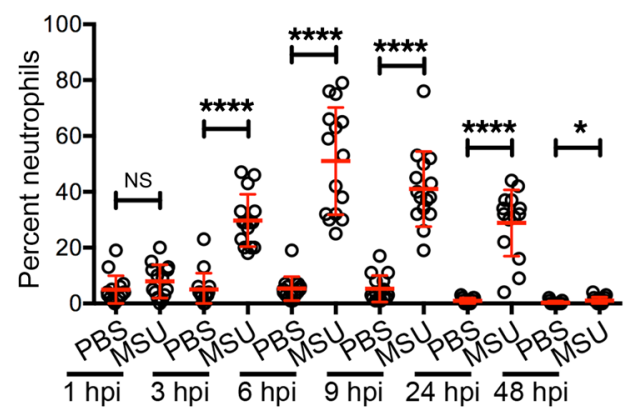

C
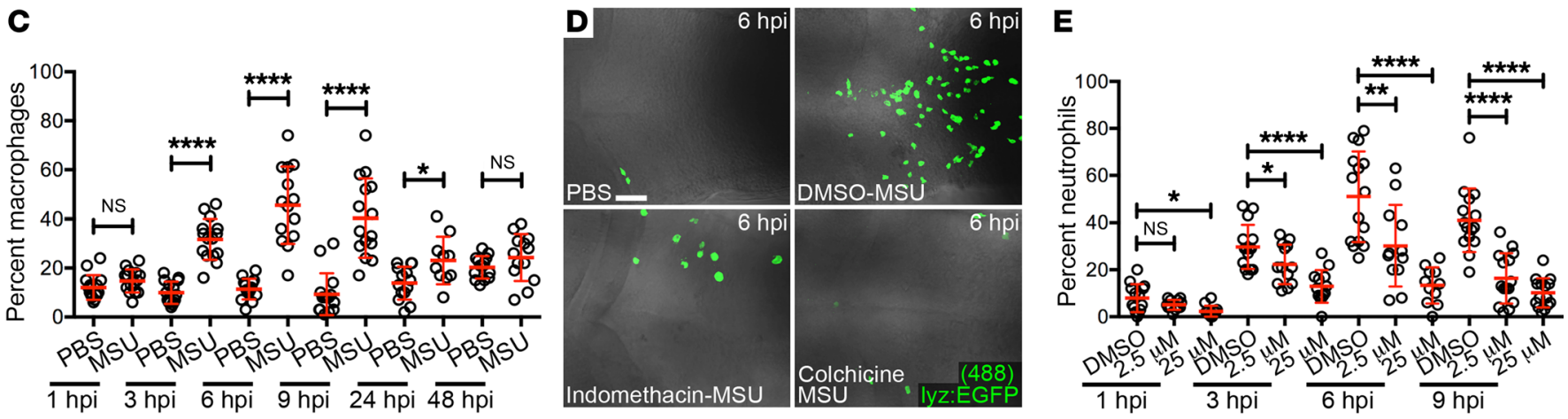

Figure 2. MSU crystals stimulate leukocyte recruitment. (A) Time-lapse imaging of neutrophil and macrophage recruitment to MSU crystals in Tg(lyz:EGFP;mpeg1:nfsB-mCherry) larvae. (B and $\mathbf{C}$ ) Temporal quantification of neutrophils (B) and macrophages (C) in the hindbrains of PBS- and MSU crystal-injected, 1\% DMSO-treated Tg(lyz:EGFP) and Tg(mpeg1:EGFP) larvae, respectively ( $n=13-15$ larvae/treatment). (D) Immunofluorescence detection of neutrophils in the hindbrains of PBS-injected and DMSO-, indomethacin- and colchicine-treated MSU crystalinjected $T g$ (lyz:EGFP) larvae. (E and $\mathbf{F}$ ) Temporal quantification of neutrophils, as detected in $\mathbf{D}$, for indomethacin (E) and colchicine $(\mathbf{F})$ treatments ( $n=13-15$ larvae/treatment). DMSO-MSU samples are the same as in B. All data were pooled from 2 independent experiments and represent the mean $\pm \mathrm{SD}$. ${ }^{*} P<0.05$, ${ }^{* *} P<0.01$, and ${ }^{* * * *} P<0.0001$, by Student's $t$ test (B and $\mathbf{C}$ ) and 1-way ANOVA with Dunnett's post hoc test (E and F). Scale bars: $50 \mu \mathrm{m}$ (A and $\mathbf{D})$.
This delivery method resulted in negligible damage to the overlying epithelium, as evidenced by $m m p 9$ expression (a marker of wounded epithelial cells) (19) being restricted to a small number of keratin $4^{+}\left(k r t 4^{+}\right)$epithelial cells (Supplemental Figure 1B). We next studied the expression of $i l 1 b$ and $i r g 1$, detected by whole-mount ISH, and Tnfa, detected by immunofluorescence within the macrophage-specific reporter line $\mathrm{Tg}$ (mpeg1:EGFP) (20). Expression of il1b and irg1 peaked at 3 hours post injection (hpi), at which point it largely marked macrophages at the hindbrain injection site (Figure 1, B-E, and Supplemental Figure 1C). Similarly, macrophage-specific Tnfa levels peaked at $3 \mathrm{hpi}$ when compared with PBS-injected controls (Figure 1, F and G). Live imaging of MSU crystal-injected $\mathrm{Tg}$ (mpeg1:nfsB-mCherry;lyz: EGFP) double-transgenic larvae (possessing mCherry ${ }^{+}$macrophages and $\mathrm{EGFP}^{+}$neutrophils) revealed that crystals were phagocytozed exclusively by macrophages, and the proportion of macrophages containing MSU crystals increased over time (Figure 2A and Supplemental Figure 1, D and E). Quantification of neutrophil and macrophage numbers within the hindbrain of MSU crystalinjected $T g(l y z: E G F P)$ and $T g(m p e g 1: E G F P)$ larvae, respectively, revealed peak numbers for both lineages at $6 \mathrm{hpi}$ (Figure 2, B and C).
To confirm that the observed activation of macrophages was not the result of a nonspecific response to a foreign particle, we also examined $i l 1 b$ and $i r g 1$ expression in response to the microinjected soluble uric acid, FluoSpheres (fluorescent microspheres readily phagocytozed by macrophages) (Supplemental Figure 1F,) and calcium pyrophosphate (CPP) crystals (another crystal that similarly activates macrophages, resulting in CPP deposition disease) (21). This analysis revealed that only CPP crystals induced a marked increase in illb and irg1 expression, albeit not to the same levels as those detected after MSU crystal injection (Supplemental Figure 1, G-J). Importantly, the conventional antiinflammatory gout flare treatments indomethacin and colchicine (22) suppressed MSU crystal-driven neutrophil recruitment in a dose-dependent fashion (Figure 2, D-F), without affecting whole-larvae neutrophil numbers (Supplemental Figure 1K).

The study of MSU crystal-driven inflammation in macrophage-depleted mice has shown that resident macrophages (already present at the site of MSU crystal injection) are the primary drivers of proinflammatory cytokine production and neutrophil recruitment (23). The temporal kinetics of macrophage activation and neutrophil recruitment in response to MSU crys- 

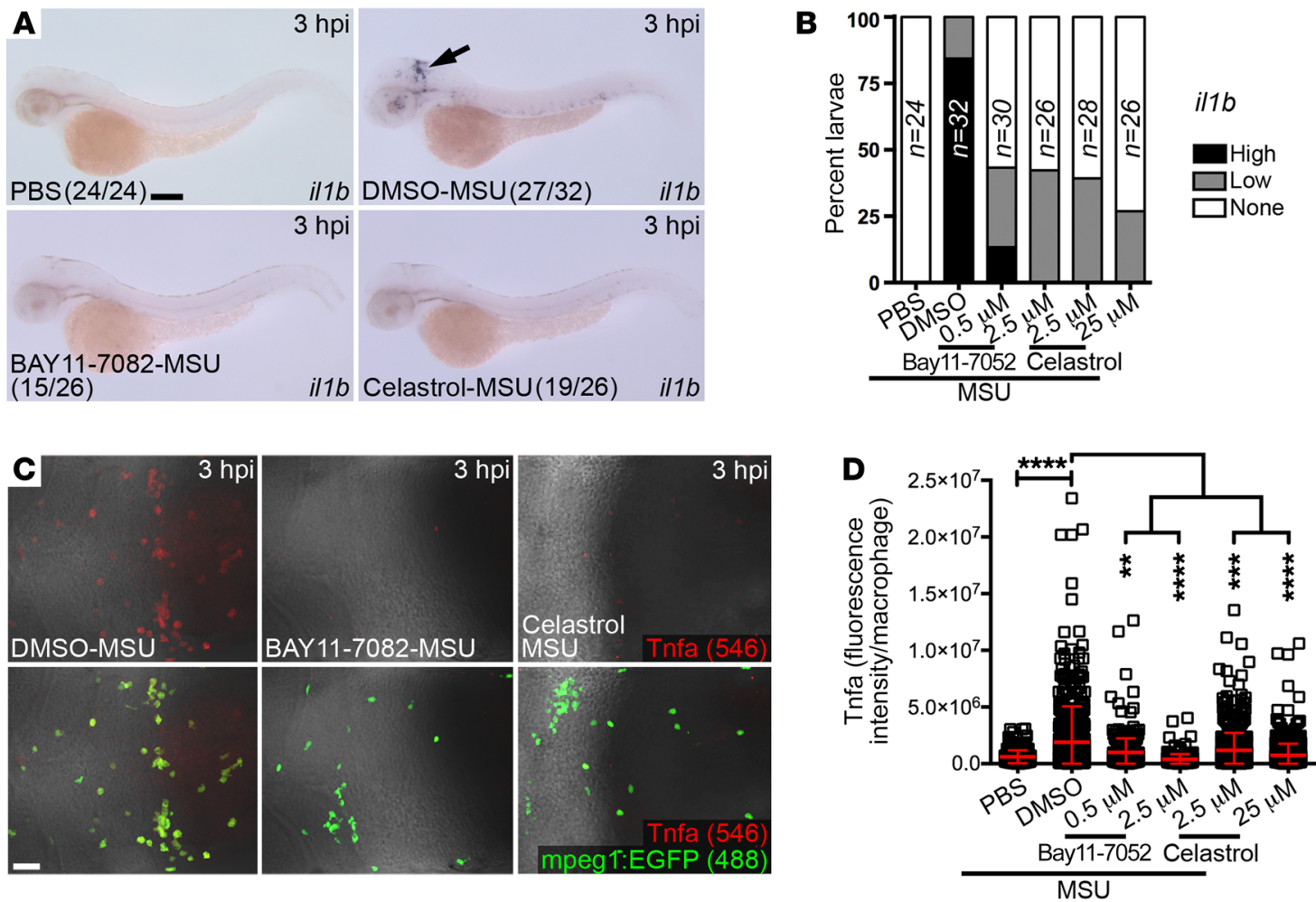

Figure 3. Macrophage activation is dependent on NF-кB signaling. (A) Expression of il1b in PBS-injected and MSU crystal-injected larvae treated with DMSO, BAY11-7082, or celastrol. Arrow indicates il1b expression in hindbrain. The numbers in parentheses indicate the frequency of larvae with the indicated phenotype.(B) Quantification of il1b expression, as detected in A. (C) Immunofluorescence of Tnfa in the hindbrains of MSU crystal-injected DMSO, BAY11-7082-, and celastrol-treated Tg(mpeg1:EGFP) larvae. (D) Quantification of Tnfa, as detected in C ( $n=15$ larvae/treatment). The DMSO-MSU sample was the same as in Figure $1 \mathrm{G}(3 \mathrm{hpi})$. Data were pooled from 2 independent experiments and represent the mean \pm SD. ${ }^{*} P<0.01,{ }^{* * *} P<0.001$, and ${ }^{* * *} P<0.0001$, by 1-way ANOVA with Dunnett's post hoc test. Scale bars: $100 \mu \mathrm{m}$ (A), $50 \mu \mathrm{m}$ (C).

tals described here (peaking at 3 and 6 hpi, respectively) supported a conserved role for macrophages in driving early neutrophil recruitment in our model. To confirm this, we used 2 strategies to specifically deplete larval macrophages. First, we took advantage of the $T g$ (mpeg1:nfsB-mCherry) transgenic line we previously used to genetically deplete macrophage-lineage cells (24). In this line, macrophages express the enzyme nitroreductase (NTR) that converts the pro-drug metronidazole (Mtz) into a cytotoxic metabolite that results in cell death. We also injected liposomal clodronate, which, following phagocytic uptake by macrophages, induces apoptosis (25). Treatment of $T g$ (mpeg1:nfsB-mCherry) larvae with 1- and 5-mM doses of Mtz caused a dose-dependent reduction in whole-larvae macrophage numbers, with approximately $30 \%$ and $80 \%$ ablation, respectively (Supplemental Figure 2, A and B), while liposomal clodronate injection resulted in $90 \%$ ablation when compared with liposome PBS-injected controls (Supplemental Figure 2, E and F). Supporting the specificity of these approaches to selectively deplete macrophages, neither treatment affected whole-larvae neutrophil numbers (Supplemental Figure 2, C and G). In addition, Mtz treatment of Tg(mpeg1:EGFP) larvae had no effect on macrophage numbers, confirming that Mtz-driven macrophage ablation in $T g$ (mpeg1:nfsB-mCherry) larvae was not the result of a toxic effect of Mtz on the macrophage lineage (Supplemental Figure 2D). Treatment of MSU crystal-injected $\mathrm{Tg}$ (mpeg1:nfsB-mCherry) larvae with Mtz led to a dose-depen- dent reduction in illb expression, while liposomal clodronate injection led to complete ablation when compared with liposome PBS-injected controls (Supplemental Figure 2, H and I). Similarly, Mtz-treated $T g$ (mpeg1:nfsB-mCherry;lyz:EGFP) larvae showed a dose-dependent reduction in neutrophil recruitment to MSU crystals (Supplemental Figure 2J), while neutrophil recruitment in liposomal clodronate-injected larvae was completely suppressed (Supplemental Figure 2K). Assessment of neutrophil recruitment to MSU crystals in $\operatorname{Tg}($ lyz:EGFP) larvae depleted of Illb (using a splice-blocking morpholino [MO], Il1b SBMO) or treated with Z-VAD-FMK (a pan-caspase inhibitor that prevents processing of pro-IL-1 $\beta$ into secreted bioactive IL-1 $\beta$ ) (26) resulted in a dose-dependent decrease in neutrophil recruitment (Supplemental Figure 3, A-D), without affecting whole-larvae neutrophil numbers (Supplemental Figure 1, K and L). When compared with control MO-injected larvae, we observed a similar inhibition of neutrophil recruitment in larvae depleted of Tnfa using independent splice (Tnfa SBMO-blocking) and translation (Tnfa ATGMO-blocking) MOs that effected a dose-dependent depletion of macrophage-specific Tnfa production (Supplemental Figure 3, A and $\mathrm{E}-\mathrm{I})$, while leaving whole-larvae neutrophil numbers unaffected (Supplemental Figure 1L).

MSU crystal-driven macrophage activation is dependent on MyD88 and NF- $\kappa B$ signaling. We next investigated whether the TLR adaptor protein myeloid differentiation primary response protein (MyD88) 

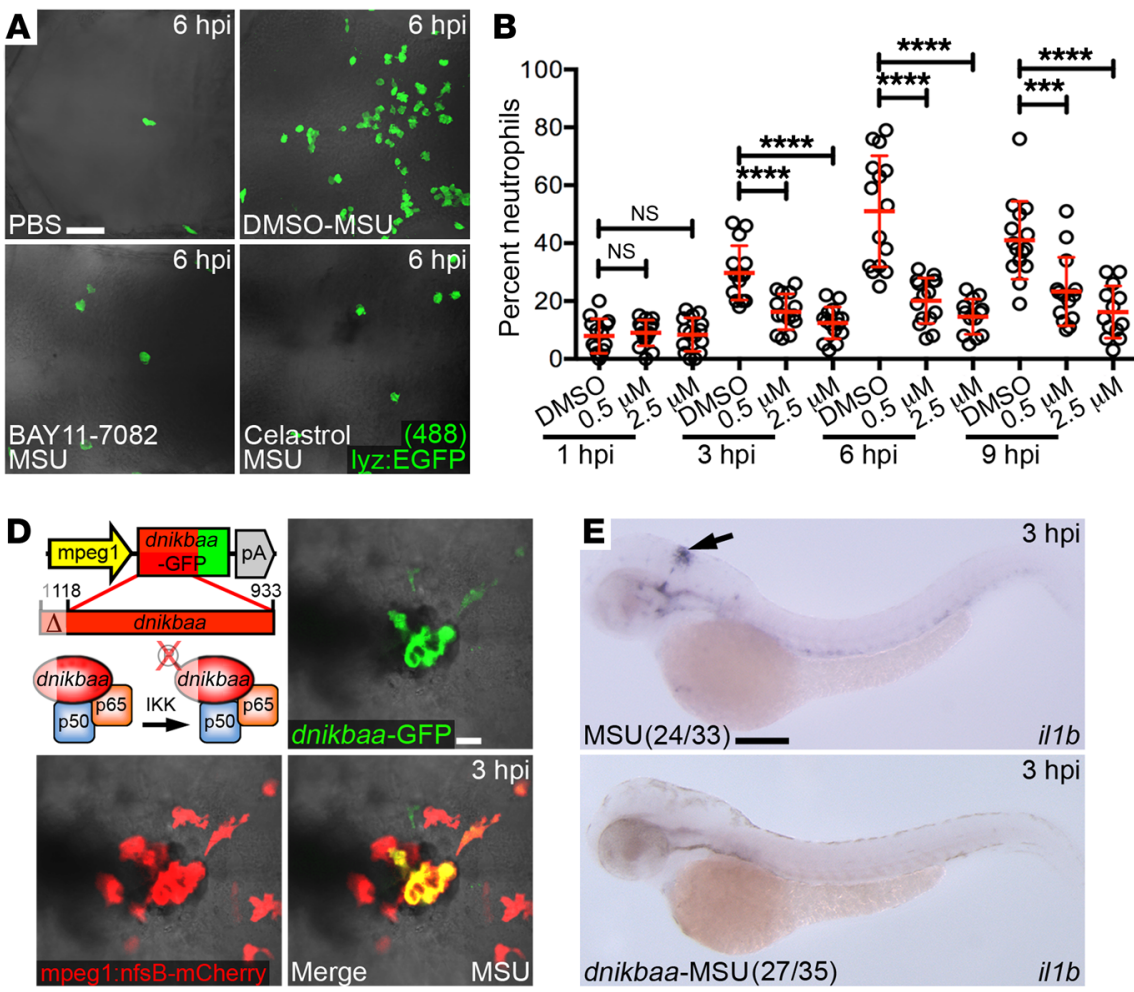
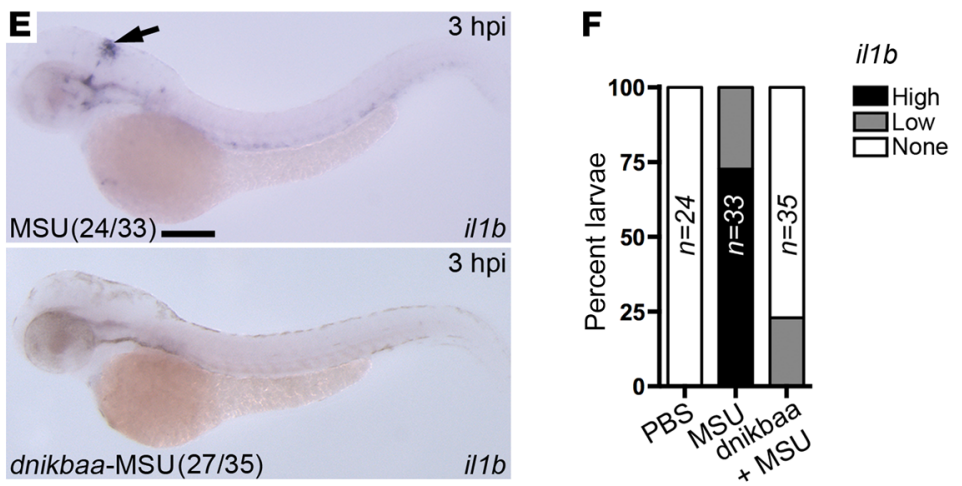

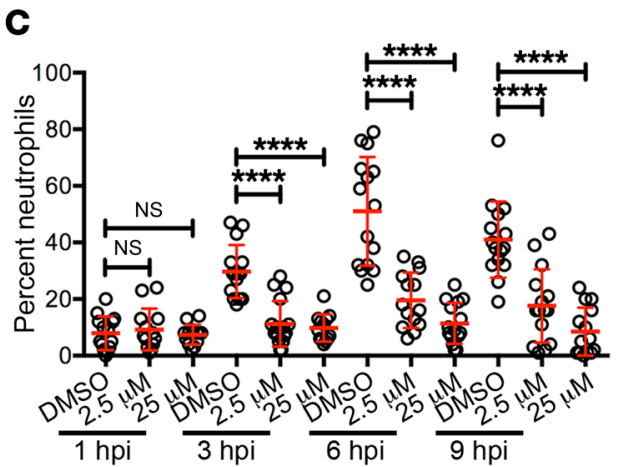

Figure 4. Neutrophil recruitment is dependent on NF-KB signaling by macrophages. (A) Immunofluorescence detection of neutrophils in the hindbrains of PBS-injected and MSU crystal-injected DMSO-, BAY11-7082-, and celastrol-treated Tg(lyz:EGFP) larvae. The DMSO-MSU image is the same as in Supplemental Figure 3B. (B and C) Temporal quantification of neutrophils, as detected in A, for BAY11-7082 (B) and celastrol (C) treatments ( $n=13-15$ larvae/treatment). The DMSO-MSU samples are the same as in Figure 2, B, E, and F, and Supplemental Figure 3D. (D) Schematic of the macrophage-specific dominant-negative IкBaa (dnikbaa) construct and expression within MSU crystal-injected Tg(mpeg1:nfsB-mCherry) larvae. (E) Expression of il1b in WT and mpeg1:dnikbaa-GFP-injected larvae following MSU crystal injection. The MSU image is the same as in Figure 1B. The numbers in parentheses indicate the frequency of larvae with the indicated phenotype. (F) Quantification of il1b expression as detected in E. The MSU sample is the same as in Figure $1 \mathrm{C}$ ( 3 hpi). The arrow marks illb expression in hindbrain. Data were pooled from 2 independent experiments and represent the mean \pm SD. ${ }^{* *} P<0.001$ and ${ }^{* * *} P<0.0001$, by 1 -way ANOVA with Dunnett's post hoc test. Scale bars: $50 \mu \mathrm{m}(\mathbf{A})$ and $100 \mu \mathrm{m}(\mathbf{E})$.

and the NF- $\kappa$ B pathway, primary mediators of MSU crystal-driven macrophage activation $(4,27)$, contributed to macrophage activation within larval zebrafish injected with MSU crystals.

Assessment of MSU crystal-driven illb expression within larvae depleted of MyD88 (using the splice-blocking MyD88 SBMO that effects a dose-dependent depletion of macrophage MyD88 protein levels [Supplemental Figure 4, A-C] and does not affect whole-larvae neutrophil numbers [Supplemental Figure 1L]), revealed a dose-dependent decrease in macrophage-specific illb expression (Supplemental Figure 4, D and E). MyD88-depleted MSU crystal-injected $\operatorname{Tg}($ mpeg1:EGFP) and $\operatorname{Tg}(l y z: E G F P)$ larvae also showed diminished macrophage-specific Tnfa production (Supplemental Figure 4, F and G) and neutrophil recruitment (Supplemental Figure $4, \mathrm{H}$ and I), respectively. To assess the contribution of NF- $\mathrm{BB}$ signaling to MSU crystal-driven macrophage activation, we used drugs that target specific components of the NF- $\kappa$ B signaling pathway. Treatment of MSU crystal-injected larvae with Bay11-7082 (an inhibitor of IкB- $\alpha$ phosphorylation) or celastrol or triptolide (inhibitors of the NF- $\kappa \mathrm{B}$ transcription factor) resulted in a dose-dependent reduction in il1b expression (Figure 3, A and B, and Supplemental Figure 5, A and B). Similar treatments of MSU crystal-injected Tg(mpeg1:EGFP) and $T g(l y z: E G F P)$ larvae caused a dose-dependent reduction in macrophage-specific Tnfa levels (Figure 3, C and D, and Supplemental Figure 5, C and D) and neutrophil recruitment (Figure 4, A-C, and Supplemental Figure 5, E and F), respectively, without affecting whole-larvae neutrophil numbers (Supplemental Figure 1K). To confirm that this requirement for NF- $\kappa B$ signaling during macrophage activation was cell autonomous, we drove expression of a dominant-negative IкBaa-GFP fusion construct (dnikbaa-GFP) specifically within macrophages. This construct encodes a truncated IкBaa (lacking the first 177 nucleotides) that cannot be phosphorylated by IкB kinase (IKK) (Figure 4D and ref. 28). We targeted dnikbaa-GFP expression to macrophages using the mpeg1 promoter (Figure 4D and ref. 20), which suppressed MSU crystal-driven macrophage-specific il1b expression (Figure 4, E and F), Tnfa production (Supplemental Figure 5, G and H), and neutrophil recruitment (Supplemental Figure 5, I and J), without affecting whole-larvae neutrophil numbers (Supplemental Figure 1L).

Macrophage activation in response to MSU crystals is dependent on JAK2/STAT3-driven expression of Irg1. We next sought to investigate a potential role for Irg1 in our zebrafish model of acute gouty inflammation.

Larvae depleted of Irg1 (using the independent SBMOs Irg1 SBMO1 and SBMO2) showed a dose-dependent reduction in MSU crystal-induced il1b expression (Figure 5, A and B, and Supplemental Figure 6, A-C). While maintaining typical numbers of 

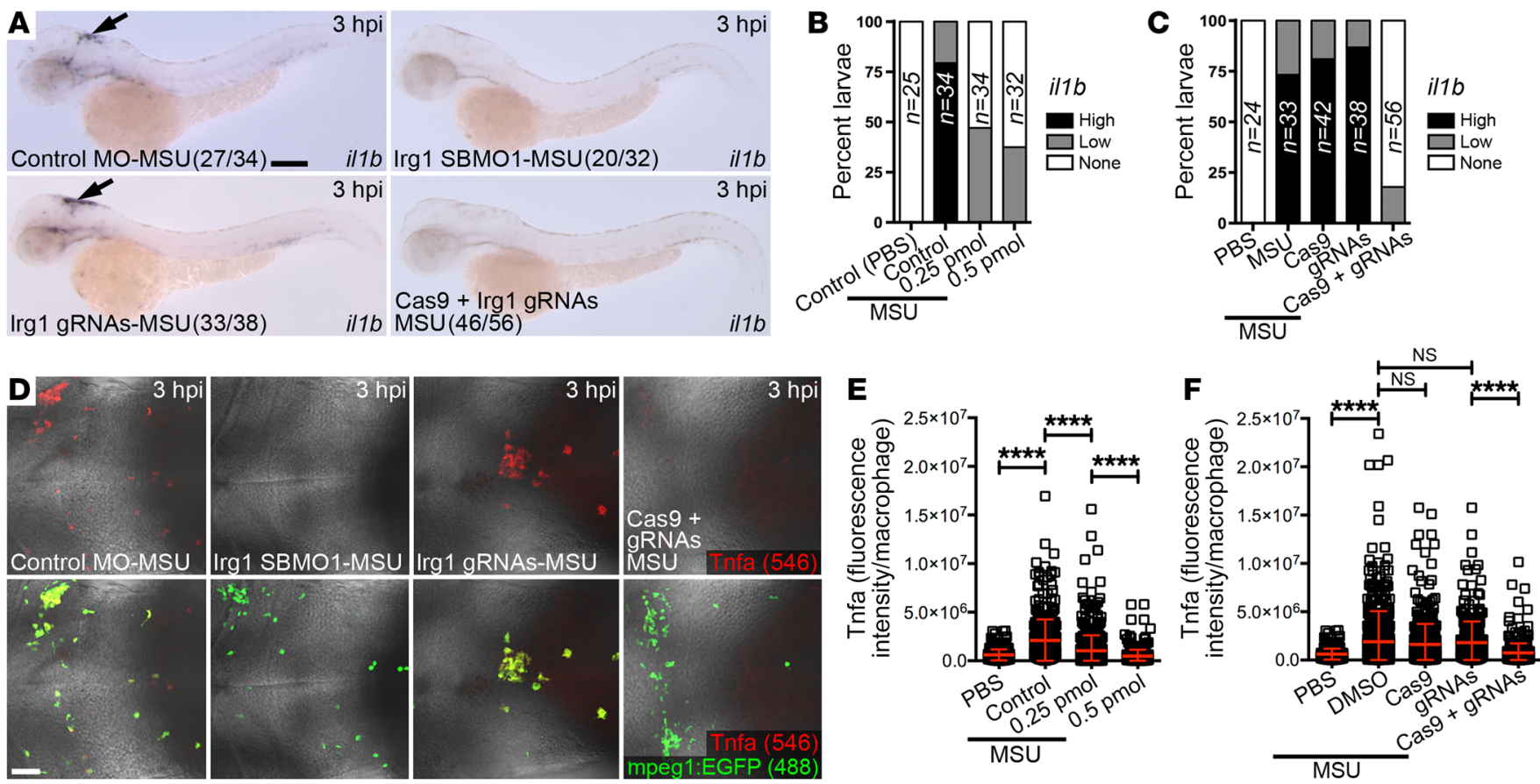

Figure 5. Irg1 contributes to MSU crystal-driven macrophage activation. (A) Expression of il1b in control MO-, Irg1 SBM01-, Irg1 gRNA-, and Cas9 plus Irg1 gRNA-injected larvae following MSU crystal injection. The numbers in parentheses indicate the frequency of larvae with the indicated phenotype. (B and C) Quantification of illb expression, as detected in A, for Irg1 SBM01-injected (B) and CRISPR-Cas9 F0 irg1 mutants (C). The MSU sample in C is the same as in Figure 1C (3 hpi) and Figure 4F. (D) Immunofluorescence of Tnfa in the hindbrains of control M0-, Irg1 SBM01-, Irg1 gRNA-, and Cas9 plus Irg1 gRNAinjected Tg (mpeg1:EGFP) larvae following MSU crystal injection. The control MO-MSU image is the same as in Supplemental Figure 3E and Supplemental Figure 4F. (E and F) Quantification of Tnfa, as detected in D, for Irg1 SBM01-injected (E) and CRISPR-Cas9 F0 irg1 mutants (F). $n=15$ larvae/treatment. The control MO-MSU sample in $\mathbf{E}$ is the same as in Supplemental Figure 3F and Supplemental Figure 4G. The DMSO-MSU sample in $\mathbf{F}$ is the same as in Figure 1G (3 hpi); Figure 3D; and Supplemental Figure 5, D and H. Data were pooled from 2 independent experiments and represent the mean \pm SD. ${ }^{* * * *} P<0.0001$, by 1-way ANOVA with Dunnett's post hoc test. Scale bars: $100 \mu \mathrm{m}$ (A) and $50 \mu \mathrm{m}$ (D).

neutrophils (Supplemental Figure 1L), MSU crystal-injected Irg1 morphants also had suppressed macrophage-specific Tnfa production and neutrophil recruitment (Figure 5, D and E, Figure 6, A and B, and Supplemental Figure 6, D-G). To confirm this result, we generated transient FO CRISPR-Cas9 irg1 mutants using 2 guide RNAs (gRNAs) that targeted exons 2 and 5 of the irg1 gene (Supplemental Figure 6H). Sequencing of individual gRNA/Cas9-injected larvae at $2 \mathrm{dpf}$ revealed mosaic excision of a large $(-2,750 \mathrm{bp})$ genomic region between the gRNA targets when compared with larvae injected with gRNAs alone (Supplemental Figure 6H). When compared with larvae injected with Cas9 only or gRNAs only, gRNA/Cas9-injected larvae had diminished MSU crystal-driven, macrophage-specific il1b expression (Figure 5, A and C) and Tnfa levels (Figure 5, D and F) and suppressed neutrophil recruitment (Figure 6, A and C), with unaltered numbers of neutrophils (Supplemental Figure 1L). Consistent with IL-8 being a critical driver of neutrophil recruitment to MSU crystals (29), Irg1-depleted larvae also expressed lower levels of $c x c l 8-l 1$ and $c x c l 8$ - $l 2$ (the zebrafish IL-8 homologs) following MSU crystal injection when compared with control MO-injected larvae (Supplemental Figure 6I). We used a MitoSOX probe to live-image macrophage-specific $\mathrm{mROS}\left(\mathrm{O}_{2}^{-}\right)$production within MSU crystal-injected Tg(mpeg1:EGFP) larvae (16) and found enhanced mROS production when compared with PBS-injected controls (Figure 6, D and E). This elevated mROS production was abolished in Irg1 morphants (Figure 6, D and E, and Supplemental Figure 6, J and K) and in FO CRISPR-Cas9 irg1 mutants (Figure 6, D and F) when compared with control MOinjected larvae and controls injected with Cas9 only or gRNAs only, respectively.

Using small-molecule inhibitors, we have previously shown that JAK2/STAT3 signaling is necessary for irg1 expression within macrophages following bacterial infection (16). Treatment of MSU crystal-injected larvae with either the STAT3 inhibitor peptide PpYLKTK-mts (STAT3 IP) or AG490 (a cell-permeable JAK2 inhibitor) resulted in a dose-dependent reduction in irg1 and il1b expression (Supplemental Figure 7, A-C). Without affecting whole-larvae neutrophil numbers (Supplemental Figure 1K), this inhibition of JAK2/STAT3 signaling also resulted in suppressed macrophage-specific Tnfa production (Supplemental Figure 7, D and E), neutrophil recruitment (Supplemental Figure 7, F and G), and macrophage-specific mROS production (Supplemental Figure 7, $\mathrm{H}$ and I) in response to MSU crystals.

MSU crystal-driven macrophage activation is dependent on FAO-fueled mROS production. We have previously shown that FAO contributes to bactericidal mROS production within infected zebrafish macrophages (16). We next studied whether FAO was necessary for MSU crystal-stimulated mROS production and whether it contributed to macrophage activation and neutrophil recruitment. 

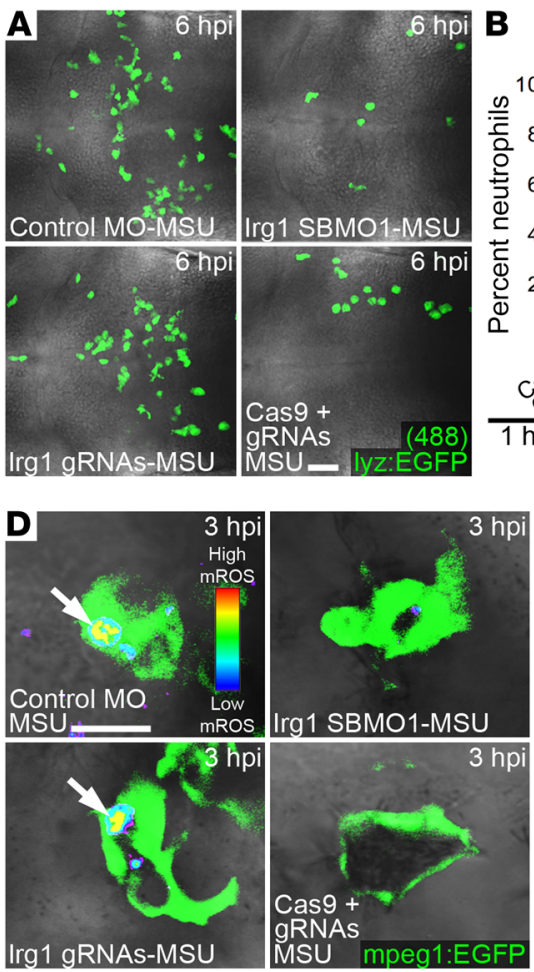
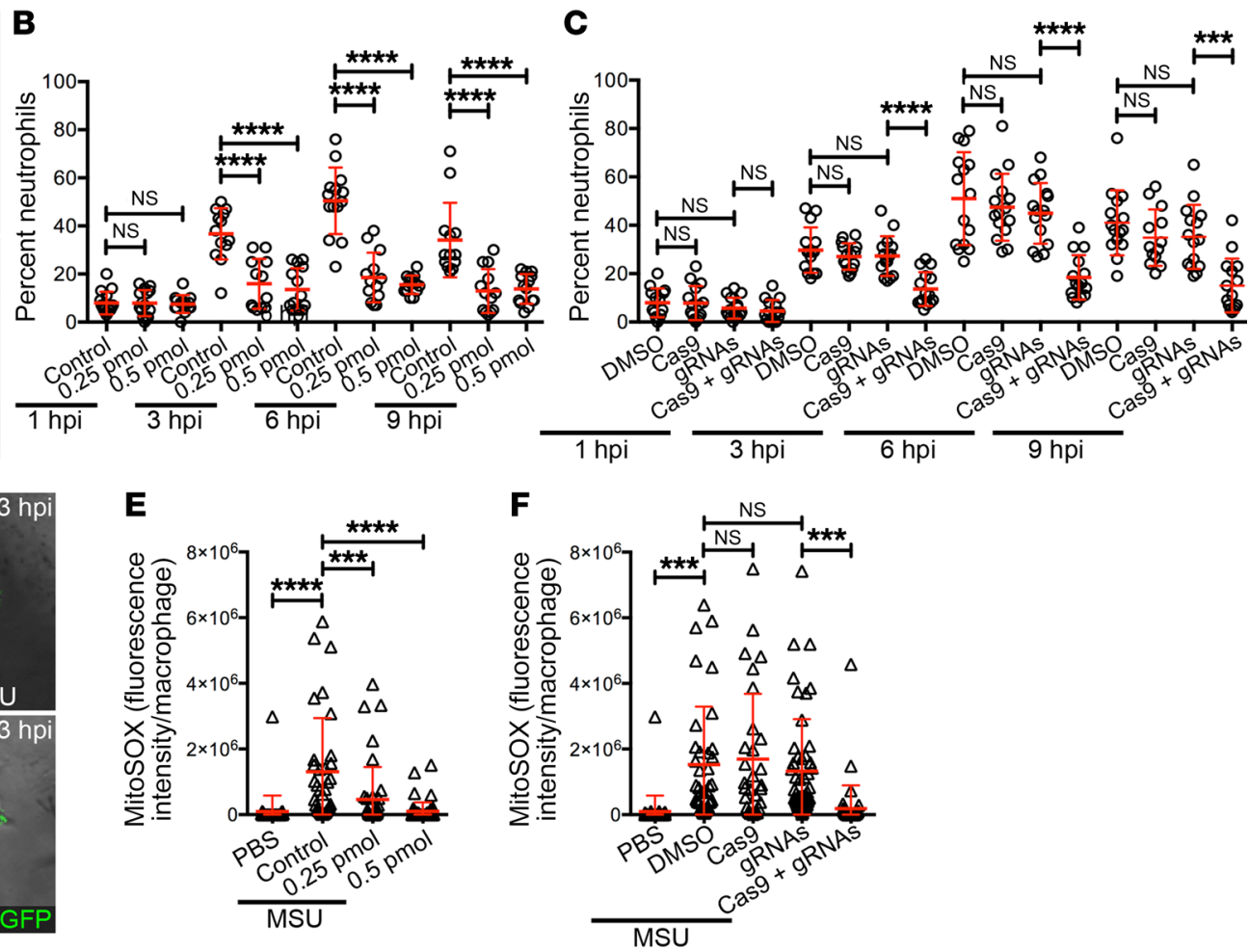

Figure 6. Irg1 contributes to MSU crystal-driven neutrophil recruitment and macrophage-specific mROS production. (A) Immunofluorescence detection of neutrophils in the hindbrains of control MO-, Irg1 SBM01-, Irg1 gRNA-, and Cas9 plus Irg1 gRNA-injected Tg(lyz:EGFP) larvae following MSU crystal injection. The control MO-MSU image is the same as in Supplemental Figure 3, B and G, and Supplemental Figure 4H. (B and C) Temporal quantification of neutrophils in the hindbrain, as detected in A, for Irg1 SBM01-injected (B) and CRISPR-Cas9 FO irg1 mutants (C). $n=13-15$ larvae/treatment. The control MO-MSU samples in B are the same as in Supplemental Figure 3, C, H, I, and Supplemental Figure 4l. The DMSO-MSU samples in C are the same as in Figure 2, B, E, and F; Figure 4, B and C; Supplemental Figure 3D; and Supplemental Figure 5, F and J. (D) Macrophage mROS production (white arrows) in the hindbrains of control MO-, Irg1 SBM01-, Irg1 gRNA-, and Cas9 plus Irg1 gRNA-injected Tg(mpeg1:EGFP) larvae following MSU crystal injection (MitoSOX signal is displayed as a heatmap, with warmer colors representing higher levels of mROS). (E and F) Quantification of macrophage-specific mROS production, as detected in $\mathbf{D}$, for Irg1 SBM01-injected (E) and CRISPR-Cas9 FO irg1 mutants (F). $n=10$ larvae/treatment. Data were pooled from 2 independent experiments and represent the mean \pm SD. ${ }^{* *} P<0.001$ and ${ }^{* * * *} P<0.0001$, by 1-way ANOVA, Dunnett's post hoc test. Scale bars: $50 \mu \mathrm{m}$ (A) and $10 \mu \mathrm{m}$ (D).

Treatment of MSU crystal-injected larvae with etomoxir, an inhibitor of carnitine palmitoyltransferase 1 (Cpt1), an enzyme essential for FAO (30), or MitoTEMPO, a mitochondrially targeted antioxidant (31), resulted in dose-dependent inhibition of macrophage-specific il1b expression (Figure 7, A and B), Tnfa production (Figure 7, C and D), and neutrophil recruitment (Figure 8, A-C), without affecting whole-larvae neutrophil numbers (Supplemental Figure 1K). These treatments also suppressed macrophage-specific mROS production (Figure 8, D and E). Given that flares of acute joint inflammation correlate with elevated serum FA levels $(11,12)$, we examined the effects of elevated serum FAs on MSU crystal-driven, macrophage-specific mROS production through acute intravenous administration of intralipid (32). Coinjection of intralipid with the fluorescent $F A$ analog BODIPY FL $\mathrm{C}_{16}$, which permits live imaging of FA transport within zebrafish larvae (33), into the circulation of $T g(k d r l: R F P)$ (34) larvae (possessing red fluorescent vasculature) revealed the immediate transport of injected lipids throughout the vasculature and progressive accumulation in the hindbrain ventricle within 1 hour (Figure 9, A and B). Similar intravenous delivery of intralipid, immediately before MSU crystal injection into $\mathrm{Tg}$ (mpeg1:EGFP) larvae, resulted in elevated macrophage-specific mROS production, but not in the pres- ence of etomoxir (Figure 9C). Furthermore, direct coinjection of the FAs C16:0 (palmitic acid) and C18:0 (stearic acid) with MSU crystals into the hindbrain ventricle of $\mathrm{Tg}$ (mpeg1:EGFP) larvae, in the presence and absence of etomoxir, similarly elevated macrophage-specific mROS production through a FAO-dependent mechanism (Supplemental Figure 8, A-C).

The contribution of $m R O S$ to MSU crystal-driven macrophage activation requires $N F-\kappa B$ signaling. Possessing an ability to diffuse between cellular compartments, $\mathrm{H}_{2} \mathrm{O}_{2}$ functions as a signaling molecule coupling $\mathrm{mROS}\left(\mathrm{O}_{2}^{-}\right)$with cellular pathways, including the NF- $\mathrm{BB}$ pathway and cytokine production $(35,36)$. We studied whether the contribution of $\operatorname{mROS}\left(\mathrm{O}_{2}^{-}\right) / \mathrm{H}_{2} \mathrm{O}_{2}$ to MSU crystaldriven macrophage activation required the NF- $\kappa \mathrm{B}$ pathway.

To examine whether microinjected $\mathrm{H}_{2} \mathrm{O}_{2}$ could rescue $\mathrm{MSU}$ crystal-stimulated macrophage activation when endogenous mROS production was suppressed (Figure 10A), we used the genetically encoded ratiometric sensor HyPer that facilitates live imaging of intracellular $\mathrm{H}_{2} \mathrm{O}_{2}$ (37). Hindbrain microinjection of $\mathrm{H}_{2} \mathrm{O}_{2}$ into $\mathrm{Hy}$ Per mRNA-injected 2-dpf larvae resulted in an elevated HyPer ratio $\left(\mathrm{HyPer}_{488} / \mathrm{HyPer}_{405}\right.$ ) within cells in the hindbrain, which was indicative of elevated intracellular $\mathrm{H}_{2} \mathrm{O}_{2}$ (Supplemental Figure 9A). Measurement of macrophage-specific HyPer ratios 
A

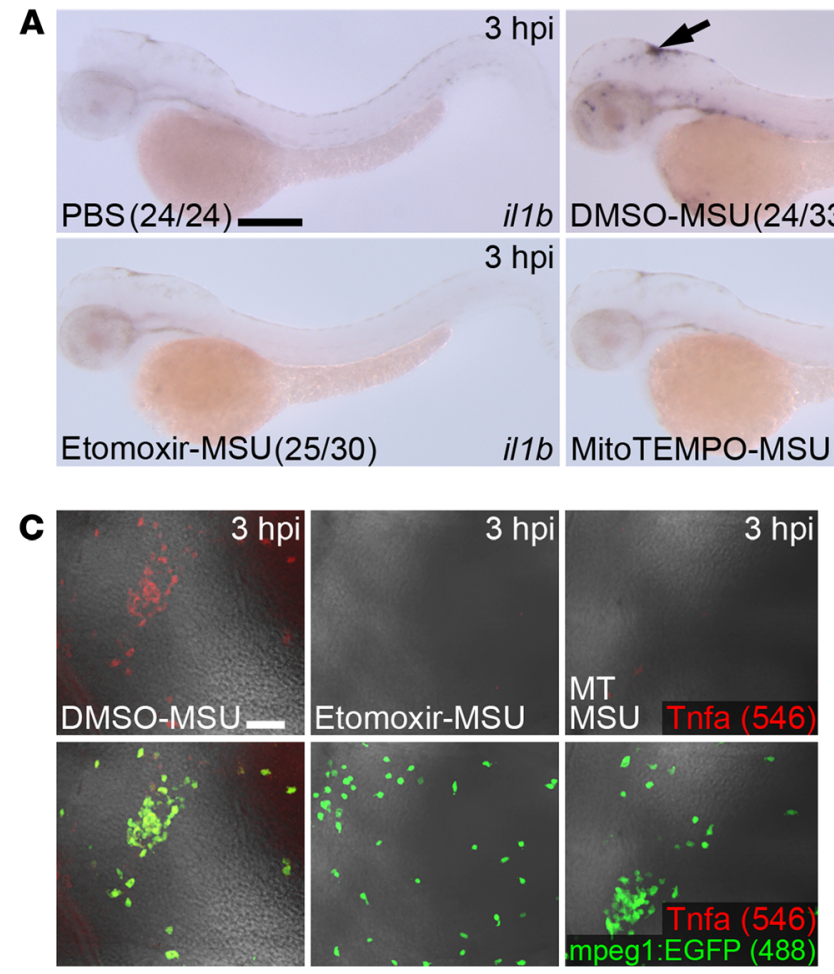

B $i l 1 b$ High $\square$ Low $\square$ None

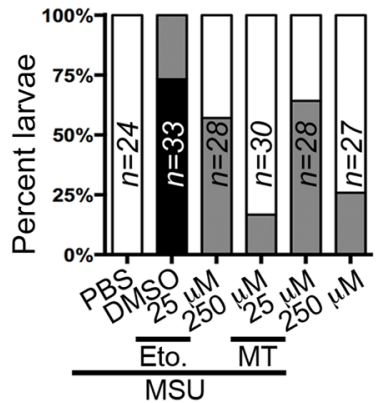

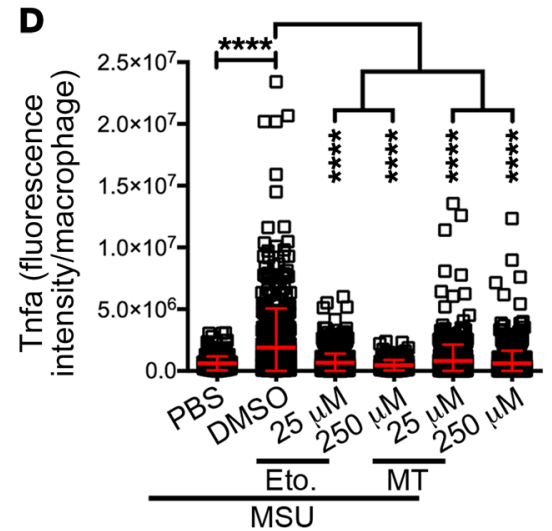

Figure 7. FAO and mROS production contributes to MSU crystal-driven macrophage activation. (A) Expression of $i / 1 b$ in PBS-injected and MSU crystal-injected DMSO-, etomoxir-, and MitoTEMPO-treated larvae. The PBS and DMSO-MSU images are the same as in Figure 1B, Supplemental Figure 1G, and Supplemental Figure 7A, respectively. Arrow indicates il1b expression in the hindbrain. The numbers in parentheses indicate the frequency of larvae with the indicated phenotype. (B) Quantification of i/1b expression, as detected in A. The DMSO-MSU sample is the same as in Supplemental Figure 7C. (C) Immunofluorescence of Tnfa in the hindbrains of MSU crystal-injected DMSO-, etomoxir- and MitoTEMPO-treated Tg(mpeg1:EGFP) larvae. The DMSO-MSU image is the same as in Supplemental Figure 5C. (D) Quantification of Tnfa, as detected in C ( $n=15$ larvae/ treatment). The DMSO-MSU sample is the same as in Figure 1G (3 hpi), Figure 3D; Figure 5F; Supplemental Figure 5, D and H; and Supplemental Figure 7E. Data were pooled from 2 independent experiments and represent the mean \pm SD. ${ }^{* * *} P<0.0001$, by 1 -way ANOVA with Dunnett's post hoc test. Scale bars: $100 \mu \mathrm{m}$ (A), $50 \mu \mathrm{m}$ (C). Eto., etomoxir; MT, MitoTEMPO.

within individual macrophages before and after $\mathrm{H}_{2} \mathrm{O}_{2}$ delivery (by injecting mpeg1:Gal4FF and UAS:HyPer Tol2 constructs that restricted HyPer expression to macrophages) revealed that microinjected $\mathrm{H}_{2} \mathrm{O}_{2}$ could diffuse into resident macrophages to increase intracellular levels of $\mathrm{H}_{2} \mathrm{O}_{2}$ (Figure 10, A-C). Injection of $\mathrm{H}_{2} \mathrm{O}_{2}$ into MSU crystal-injected larvae treated with either STAT3 IP, AG490, MitoTEMPO, or etomoxir (to inhibit mROS production in macrophages) was sufficient to rescue macrophage-specific illb expression (Figure 10, D and E, and Supplemental Figure 9, B and C), Tnfa production (Figure 11, A and B, and Supplemental Figure 9, D and E), and neutrophil recruitment (Figure 11, C-E, and Supplemental Figure 10, $\mathrm{A}-\mathrm{C}$ ) in a dose-dependent manner. Similar delivery of $\mathrm{H}_{2} \mathrm{O}_{2}$ did not effect the rescue of macrophage activation or neutrophil recruitment when injected into MitoTEMPO-treated larvae expressing the mpeg1:dnikbaa-GFP construct (Figure 10, D and E, and Figure 11, A-E). Of note, despite the fact that $\mathrm{H}_{2} \mathrm{O}_{2}$ provides an endogenous gradient that attracts wound-responding neutrophils (37), injection of $\mathrm{H}_{2} \mathrm{O}_{2}$ alone did not attract neutrophils to the hindbrain injection site (Supplemental Figure 10D).

Drugs that inhibit Irg1-dependent mROS production suppress macrophage activation and neutrophil recruitment to $M S U$ crystals. Through simple immersion in drug, larval zebrafish permit a live, whole vertebrate bioassay approach to assess drug activity in a high-content fashion (38). To uncover new therapeutics that suppress macrophage-driven inflammation, we performed a drug-repositioning screen to identify drugs that inhibit macrophage-specific irg1 expression. Using the Prestwick Chemical library containing 1,280 drugs, we identified 3 drugs (chrysin, piperlongumine, and camptothecin) that strongly suppressed irg1 expression within LPS-injected 2-dpf zebrafish larvae (Supplemental Figure 11A). We studied the therapeutic effects of these drugs in our larval zebrafish model of acute gouty inflammation and found that they also inhibited MSU crystal-driven expression of irg1 (Supplemental Figure 11, B and C) and expression of an irg1:EGFP transgene that provides a live readout of macrophage activation within Tg(irg1:EGFP;mpeg1:nfsB-mCherry) larvae (Supplemental Figure 11, D-G and ref. 39). Treatment of MSU crystal-injected larvae with chrysin, piperlongumine, or camptothecin also suppressed macrophage-specific il1b expression (Figure 12, A and B), Tnfa production (Figure 12, C and D), and neutrophil recruitment (Figure 13, A-D), without affecting whole-larvae neutrophil numbers (Supplemental Figure 1K), and suppressed macrophage-specific mROS production (Figure 13, E and F). 

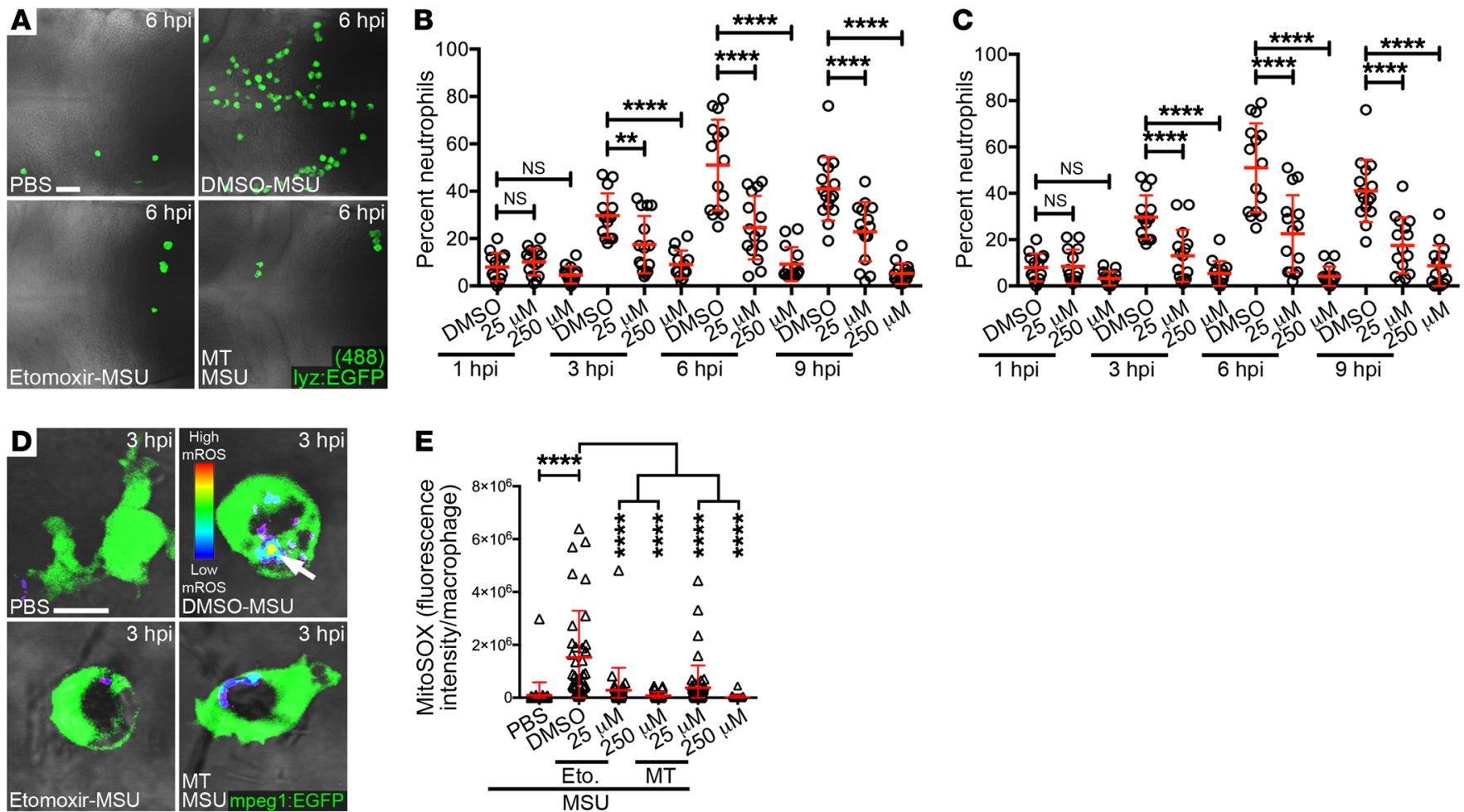

Figure 8. FAO and mROS production contributes to MSU crystal-driven neutrophil recruitment. (A) Immunofluorescence detection of neutrophils in the hindbrains of MSU crystal-injected DMSO-, etomoxir-, and MitoTEMPO-treated Tg(lyz:EGFP) larvae. The DMSO-MSU image is the same as in Supplemental Figure 7F. (B and C) Temporal quantification of neutrophils, as detected in A, for etomoxir (B) and MitoTEMPO (C) treatments ( $n=13-15$ larvae/treatment). The DMSO-MSU samples are the same as in Figure 2, B, E, and F; Figure 4, B and C; Figure 6C; Supplemental Figure 3D; Supplemental Figure 5, F and J; and Supplemental Figure 7C. (D) Macrophage mROS production (white arrow) in the hindbrains of MSU crystal-injected DMSO-, etomoxir-, and MitoTEMPO-treated Tg(mpeg1:EGFP) larvae (MitoSOX signal is displayed as a heatmap, with warmer colors representing higher levels of mROS). The PBS image is the same as in Supplemental Figure 7H. (E) Quantification of macrophage-specific mROS production, as detected in $\mathbf{D}$ ( $n=10$ larvae/treatment). The DMSO-MSU sample is the same as in Figure 6F and Supplemental Figure 7I. Data were pooled from 2 independent experiments and represent the mean $\pm \mathrm{SD}$. ${ }^{* *} P<0.01$ and ${ }^{* * *} P<0.0001$, by 1-way ANOVA with Dunnett's post hoc test. Scale bars: $50 \mu \mathrm{m}$ (A) and $10 \mu \mathrm{m}$ (D).

FAO-fueled mROS augment MSU crystal-driven expression of IL1B and TNFA within human THP-1 cells, and drugs that inhibit IRG1 expression suppress this activation and neutrophil recruitment in a mouse model of acute gouty inflammation. We next investigated whether this immunometabolic mechanism of macrophage activation operated within human monocytic THP-1 cells, a widely accepted in vitro model of acute gouty inflammation that was fundamental to uncovering the central importance of the NLRP3 inflammasome in gout (10). MSU crystal-driven expression of both $I L 1 B$ and TNFA was greatly augmented in PMA-stimulated THP-1 cells in the presence of C18:0 (Figure 14, A and B). This elevated expression was, at least in part, dependent on NF- $\mathrm{\kappa B}$ signaling, mROS, and FAO production, as evidenced by the significantly reduced expression of $I L 1 B$ and TNFA following BAY117082, celastrol, triptolide, MitoTEMPO, and etomoxir treatments (Figure 14, A and B). Consistent with their ability to suppress IRG1 expression (Figure 14C), chrysin, piperlongumine, and camptothecin treatments also inhibited this $\mathrm{FAO} / \mathrm{mROS}$-driven expression of IL1B and TNFA in THP-1 cells costimulated with MSU crystals and C18:0 (Figure 14, A and B). Costimulation of THP-1 cells with MSU crystals and C18:0 also augmented mROS production compared with stimulation with MSU crystals alone (Figure 14, D and E), but not in the presence of MitoTEMPO, confirming the spec- ificity of the MitoSOX probe for the detection of mROS in THP-1 cells. This increase in mROS production was also sensitive to etomoxir treatment and did not occur in the presence of the IRG1inhibiting drugs chrysin, piperlongumine, or camptothecin (Figure 14, D and E). Next, we examined whether Irg1 was expressed in murine monocytes stimulated with MSU crystals using the MSU crystal-induced peritonitis model of acute gouty inflammation, a well-established model in which resident macrophages initiate and drive neutrophil recruitment (23). We observed that the peritoneal monocytes, isolated following intraperitoneal injection of MSU crystals, expressed Irg1 with temporal kinetics identical to those for Il1b and Tnfa (Figure 14, F-H). Finally, we assessed the effects of chrysin, piperlongumine, and camptothecin in a murine air pouch model of acute gouty inflammation (in which MSU crystals are injected into an air pouch that morphologically resembles synovium; ref. 40) and found that chrysin and piperlongumine treatments effected a dose-dependent reduction in neutrophil recruitment (Figure 14, I and J).

\section{Discussion}

We developed a unique larval zebrafish model of MSU crystaldriven acute inflammation that provided a window for directly observing the macrophage response to MSU crystals within a 
A
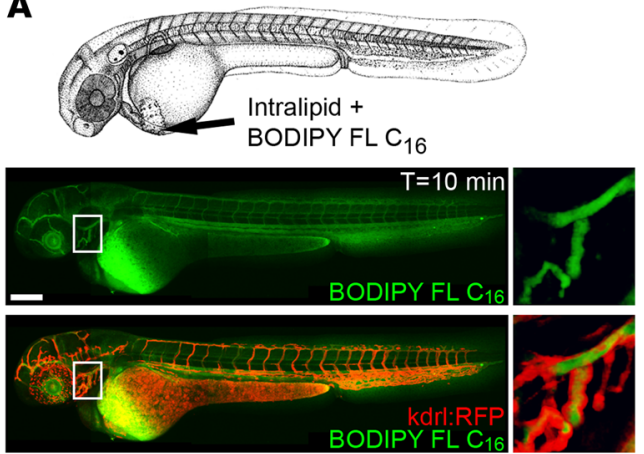

B

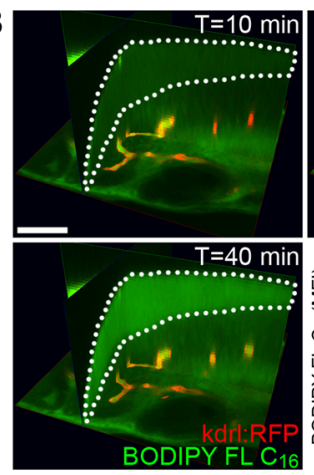

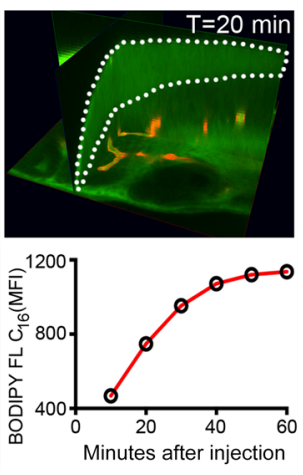

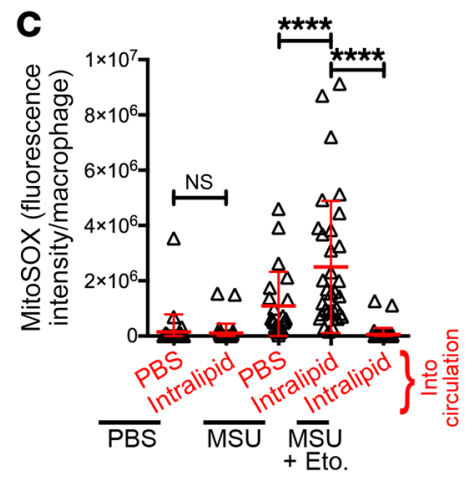

Figure 9. Intravenous delivery of intralipid elevated MSU crystal-driven macrophage-specific mROS production through a FAO-dependent mechanism. (A) Live imaging of a $\mathrm{Tg}(\mathrm{kdrl}: R F P)$ larva following injection of intralipid, supplemented with BODPIY FL $\mathrm{C}_{16}$, into the sinus venosus (insets show magnified views of the boxed areas). (B) $X / Y / Z$ views of hindbrain of a $T g(k d r l: R F P)$ larva, as treated in $\mathbf{A}$, showing accumulation of BODPIY FL $C_{16}$ in the hindbrain ventricle (dashed lines) and temporal quantification of BODPIY FL $C_{16}$ MFI in the hindbrain. (C) Quantification of macrophage-specific mROS production (MitoSOX signal) in the hindbrains of PBS- or MSU crystal-injected Tg (mpeg1:EGFP) larvae following delivery of PBS or intralipid into the circulation ( $n=10$ larvae/ treatment). Data for $\mathbf{C}$ were pooled from 2 independent experiments. All data represent the mean \pm SD. ${ }^{* * *} P<0.0001$, by 1-way ANOVA with Dunnett's post hoc test. Scale bars: $100 \mu \mathrm{m}(\mathbf{A})$ and $50 \mu \mathrm{m}(\mathbf{B})$.

completely intact host. Importantly, the larval zebrafish innate immune cell response to MSU crystals was remarkably conserved compared with that described in mammalian systems, including the kinetics of macrophage activation and neutrophil recruitment and the sensitivity of this model to conventional antiinflammatory gout treatments. Using the live imaging potential of this model, we provide mechanistic insights into acute gouty inflammation by showing that FAO-fueled mROS production contributes to macrophage activation by promoting NF- $\mathrm{kB}-$ driven production of Il1 b and Tnfa that drives neutrophil recruitment (Supplemental Figure 12). In support of the translation of these findings to human macrophages, we further show that C18:0 augments MSU crystal-driven, NF- $\mathrm{KB}$-dependent expression of IL1B and TNFA in THP-1 cells through FAO and mROS production and that C18:0 can elevate THP-1 cell mROS production in response to MSU crystals, but not following drug-mediated blockade of IRG1 expression and FAO. We also provide evidence that our larval zebrafish model has utility as a drug discovery platform for identifying new antiinflammatory therapeutics to suppress MSU crystal-driven inflammation. Previous studies have revealed that FAs can promote the proinflammatory phenotype of macrophages, in part through activation of NF- $\mathrm{KB}$ signaling (41). Furthermore, flares of acute joint inflammation in individuals with gout correlate with elevated serum FA levels $(11,12)$, and the release of FAs into the circulation is a common metabolic consequence of a number of risk factors predisposing patients to flares (e.g., excessive alcohol and food consumption) (42). Of note, stearic acid has been shown to directly augment MSU crystal-stimulated IL-1 $\beta$ production by human PBMCs and murine macrophages through a TLR2/caspase 1-dependent mechanism (13). Our work reveals a requirement for FAO during MSU crystal-driven mROS, IL- $1 \beta$, and TNF- $\alpha$ production and provides mechanistic insights into how FAs contribute to macrophage activation during acute gouty inflammation.

Macrophages display remarkable plasticity with respect to their metabolic mode and can generate mROS through different mechanisms, including reverse electron transport $(43,44)$. Until recently, FAO was thought to be the primary metabolic mode of antiinflammatory macrophages, while aerobic glycolysis was a driver of the proinflammatory state (43). It now appears that this dichotomy is an oversimplification and that macrophage metabolic modes, much like their phenotypes, are heterogeneous and very context dependent (45). As an example, when investigating single-nucleotide variations in a gene of unknown function that is associated with increased risk of juvenile idiopathic arthritis, it was discovered that the encoded protein (subsequently called the FA metabolism immunity nexus [FAMIN]) regulates mROS and IL-1 $\beta$ production in proinflammatory macrophages through a FAO-dependent mechanism (31). Our work supports the emerging role of FAO during macrophage activation and highlights the importance of examining macrophage activation in the context of an intact microenvironment, where factors that help regulate the plasticity of macrophage function, such as FAs, are present. Most studies into metabolic reprogramming of macrophages have been restricted to in vitro techniques using limited inflammatory stimuli (largely LPS) (45). A current challenge for the field of immunometabolism is to study how macrophage metabolism is altered in response to alternative stimuli (such as MSU crystals) in vivo and at the single-cell level and how these changes influence macrophage function and disease pathogenesis (45). This study highlights the utility of larval zebrafish as a model system to help address this challenge.

In response to LPS, Irg1-derived itaconic acid has been shown to suppress the production of IL- $1 \beta$, but not TNF- $\alpha$, in bone marrow-derived macrophages by inhibiting succinate dehydrogenase (SDH), leading to elevated intracellular levels of succinate (46). In contrast, inhibition of Irg1 activity suppresses ROS, IL-1 1 , and TNF- $\alpha$ production in human and murine epithelial cells and reduces immune cell recruitment in a model of respiratory syncytial virus infection (47). Further supporting a proinflammatory role for Irg1, a study using an experimental model of peripheral arterial disease showed that a reduction in Irg1-drived itaconic acid promotes M2 polarization in macrophages, while elevated Irg1-drived itaconic acid indicates an M1 phenotype (48). 
A MSU crystals

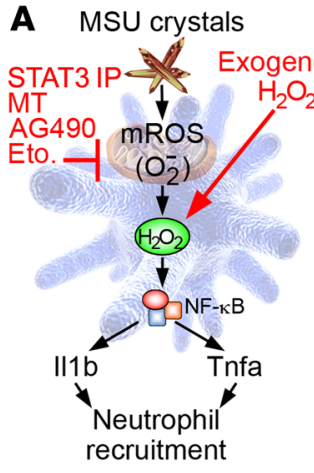

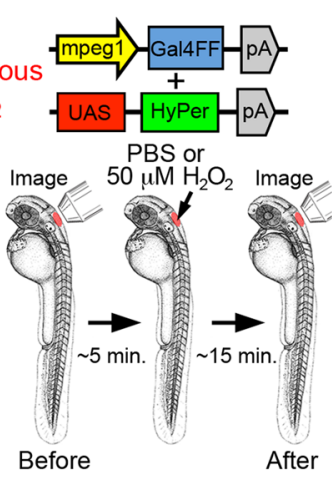
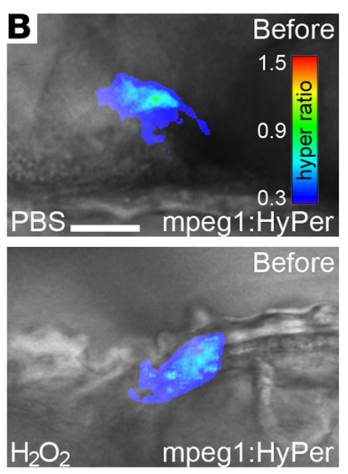
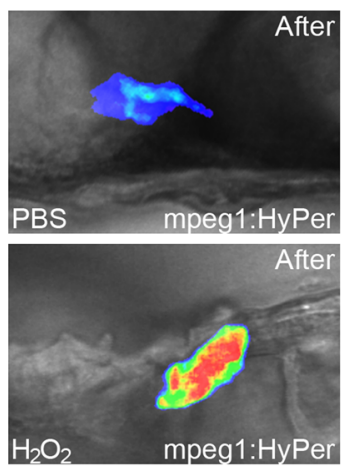

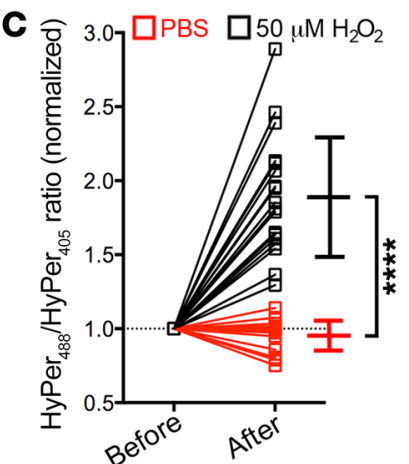

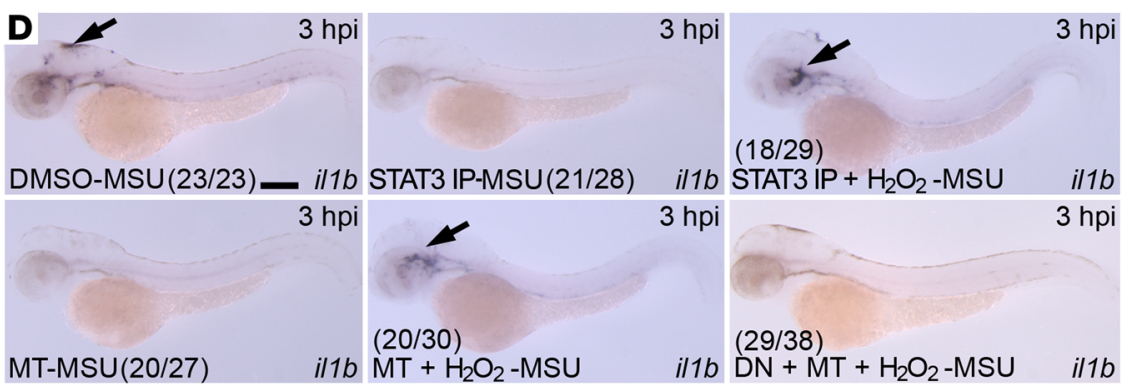

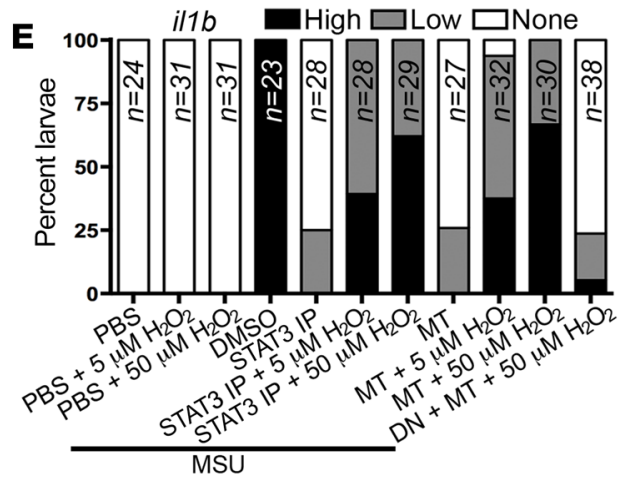

Figure 10. Exogenous $\mathrm{H}_{2} \mathrm{O}_{2}$ can rescue MSU crystal-driven il1b expression following endogenous mROS depletion. (A) Schematic of $\mathrm{H}_{2} \mathrm{O}_{2}$ rescue strategy (following STAT3 IP-, MitoTEMPO-, AG490-, and etomoxir-mediated endogenous mROS depletion) and constructs for live imaging of macrophage $\mathrm{H}_{2} \mathrm{O}_{2}$ levels. (B) Ratiometric HyPer imaging (488/405 nm ratio is displayed as a heatmap, with warmer colors representing higher $\mathrm{H}_{2} \mathrm{O}_{2}$ levels) of $\mathrm{H}_{2} \mathrm{O}_{2}$ in single hindbrain macrophages from mpeg1:Cal4FF/UAS:HyPer-injected larvae before and after injection of PBS or $50 \mu \mathrm{M} \mathrm{H}_{2} \mathrm{O}_{2}$. (C) Quantification of HyPer ratios as detected in B, measured as normalized maximum 488/405 nm ratios within individual macrophages ( $n=4$ larvae/ treatment). (D) Expression of il1b in MSU crystal-injected larvae treated with DMSO, STAT3 IP (125 $\mu$ M), MitoTEMPO (250 $\mu$ M) (with and without coinjection of $\left.50 \mu \mathrm{M} \mathrm{H}_{2} \mathrm{O}_{2}\right)$, or dnikbaa (DN) with MitoTEMPO $(250 \mu \mathrm{M})$ plus $\mathrm{H}_{2} \mathrm{O}_{2}(50 \mu \mathrm{M})$. Arrows mark illb expression in hindbrain. The numbers in parentheses represent the frequency of larvae with the indicated phenotype. (E) Quantification of illb expression, as detected in $\mathbf{D}$. Data were pooled from 2 independent experiments and represent the mean \pm SD. ${ }^{* * *} P<0.0001$, by Student's $t$ test. Scale bars: $10 \mu \mathrm{m}$ (B) and $100 \mu \mathrm{m}$ (D).

In addition, when suppressing macrophage activation through inhibition of the catabolic enzyme branched-chain aminotransferase (BCAT), decreased Irg1 and itaconic acid levels contribute to a reduction in proinflammatory gene expression (49). Here, we show that, in response to MSU crystals, Irg1 is necessary for an adaptive increase in mROS levels that in turn promotes IL-1 $\beta$ and TNF- $\alpha$ production.

By exploiting our zebrafish model of acute gouty inflammation, we identified 3 drugs - chrysin, piperlongumine, and camptothecin - that inhibited macrophage-specific mROS production, illb expression, and Tnfa production and suppressed neutrophil recruitment in response to MSU crystals. Each of these drugs have well-defined antineoplastic activities that are explained, at least in part, by their ability to inhibit STAT3 phosphorylation (50-52), which is consistent with our data showing the ability of these drugs to suppress JAK2-STAT3-dependent irg1 expression in zebrafish macrophages. We also show that 2 of these drugs, chrysin and piperlongumine, inhibited neutrophil recruitment in a mouse model of MSU crystal-driven inflammation. Given that the activity of camptothecin was conserved with THP-1 cells, we predict that the lack of efficacy of camptothecin in the air pouch model may have been the result of its documented poor solubility and bioavailability in vivo (53). Using one of the many analogs of camptothecin, which have been developed to enhance its bioavailability, may result in improved in vivo efficacy in this model (54). Collectively, this work extends the potential utility of chrysin and piperlongumine as new antiinflammatory agents to manage acute gouty inflammation. Further clinical studies will be necessary to determine whether the activities of these drugs translate to human patients with gout. The targeting of metabolic reprogramming to modulate macrophage function is emerging as a therapeutic strategy for a number of diseases including obesity-associated diabetes, cancers, and cardiovascular disease (55). As such, we predict that drugs that target this process in MSU crystal-stimulated macrophages may have therapeutic utility in other diseases. In support of this, chrysin and piperlongumine are both primarily known for their antineoplastic activities, and chrysin has been shown to limit inflammation in obese mice through suppression of the M1 macrophage phenotype and support of M2 polarization (56).

In summary, we uncovered a metabolic mechanism controlling MSU crystal-driven macrophage activation that is dependent on FAO-fueled production of mROS. Targeting this mechanism holds promise as a strategy to alleviate the debilitating inflammation triggered by MSU crystals and may also have utility in other macrophage-driven diseases. 

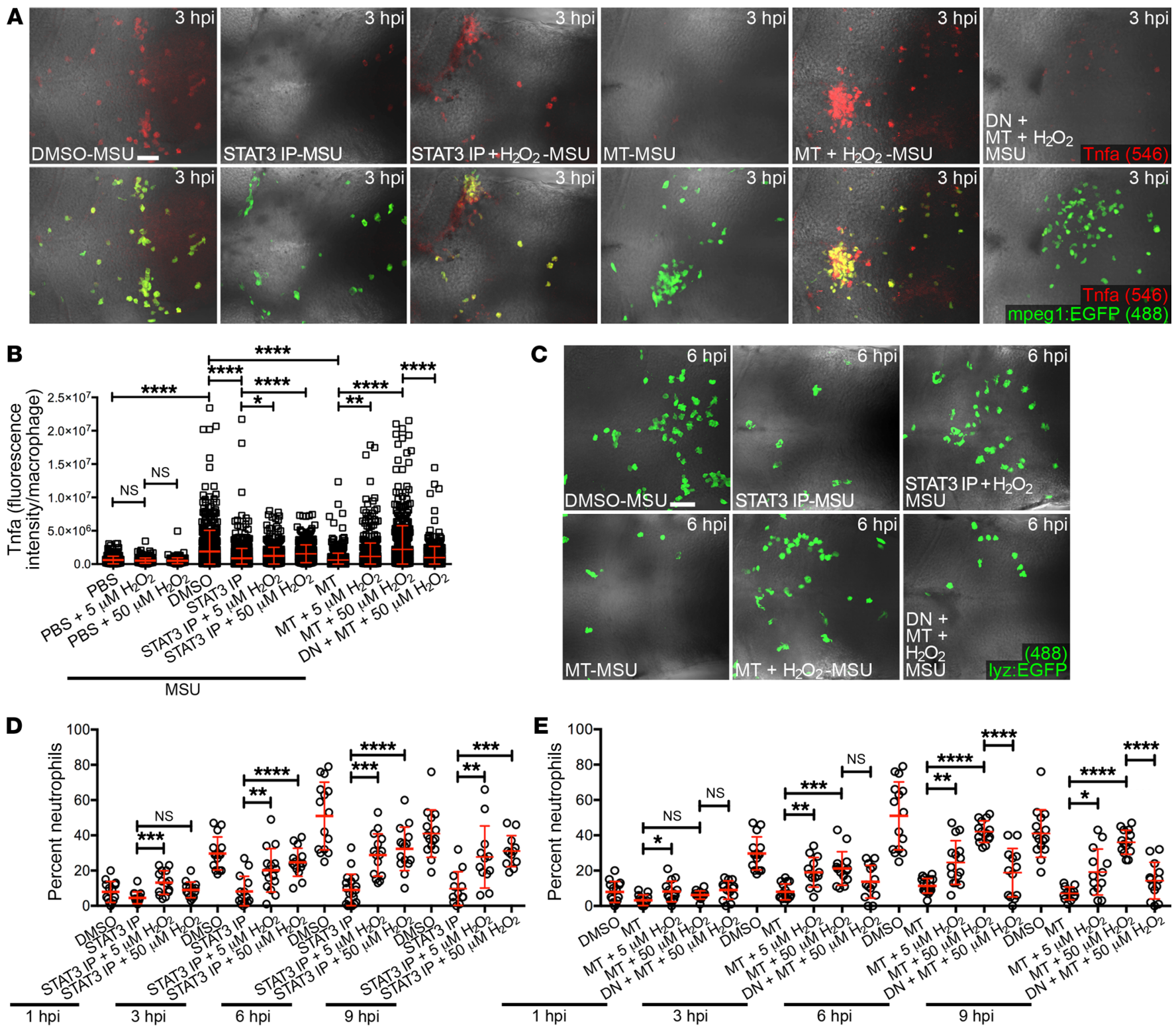

Figure 11. Exogenous $\mathrm{H}_{2} \mathrm{O}_{2}$ can rescue MSU crystal-driven macrophage-specific Tnfa production and neutrophil recruitment following endogenous mROS depletion. (A) Immunofluorescence of Tnfa in the hindbrains of MSU crystal-injected Tg(mpeg1:EGFP) larvae treated with DMSO, STAT3 IP (125 $\mu \mathrm{M})$, MitoTEMPO $(250 \mu \mathrm{M})$ (with and without coinjection of $\left.50 \mu \mathrm{M} \mathrm{H}_{2} \mathrm{O}_{2}\right)$, or dnikbaa with MitoTEMPO $(250 \mu \mathrm{M})$ plus $50 \mu \mathrm{M} \mathrm{H}_{2} \mathrm{O}_{2}$. The DMSO-MSU image is the same as in Figure 3C, Supplemental Figure 5C, and Supplemental Figure 7D. (B) Quantification of Tnfa, as detected in A ( $n=15$ larvae/treatment). The DMSO-MSU sample is the same as in Figure 1C (3 hpi); Figure 3D; Figure 5F; Figure 7D; Supplemental Figure 5, D and H; and Supplemental Figure 7E. (C) Immunofluorescence detection of neutrophils in the hindbrains of MSU crystal-injected Tg(lyz:ECFP) larvae treated with DMSO, SAT3 IP (125 $\mu$ M), MitoTEMPO $(250 \mu \mathrm{M})$ (with and without coinjected $50 \mu \mathrm{M} \mathrm{H}_{2} \mathrm{O}_{2}$ ), or dnikbaa with MitoTEMPO $(250 \mu \mathrm{M})$ plus $50 \mu \mathrm{M} \mathrm{H} \mathrm{O}_{2}$. The DMSO-MSU image is the same as in Figure 4A; Supplemental Figure 3B; and Supplemental Figure 5E. ( $D$ and E) Quantification of neutrophils, as detected in C, for STAT3 IP and $\mathrm{H}_{2} \mathrm{O}_{2}$ treatments (D) and MitoTEMPO, $\mathrm{H}_{2} \mathrm{O}_{2}$, and dnikbaa treatments (E) ( $n=13-15$ larvae/treatment). The DMSO-MSU samples are the same as in Figure 2, B, E, and F; Figure 4, B and C; Figure 6C; Figure 8, B and C; Supplemental Figure 3D; Supplemental Figure 5, F and J; Supplemental Figure 7C. Data were pooled from 2 independent experiments. Data represent the mean $\pm \mathrm{SD} .{ }^{*} P<0.05,{ }^{* *} P<0.01,{ }^{* *} P<0.001$, and ${ }^{* * * *} P<0.0001$, by 1-way ANOVA with Dunnett's post hoc test. Scale bars: $50 \mu \mathrm{m}$ (A and $\mathbf{C})$.

\section{Methods}

Zebrafish maintenance. Zebrafish (Danio rerio) embryos were obtained from natural spawnings and raised at $28^{\circ} \mathrm{C}$ in E3 medium supplemented with $0.003 \%$ phenylthiourea (PTU) to inhibit pigmentation. WT AB zebrafish were obtained from the Zebrafish International Resource Center (ZIRC). The Tg(mpeg1:Gal4FF) $)^{g 25}$ (20) and $\operatorname{Tg}$ (UAS-E1b:nfsB-mCherry) ${ }^{\mathrm{c} 264}$ (57) lines [herein referred to as
Tg(mpeg1:nfsB-mCherry) ], Tg(lyz:EGFP $)^{N z 117}$ (58), Tg(mpeg1:EGFP) $)^{g 122}$ (20), $T g(\operatorname{irg1:EGFP})^{N Z 4}(39)$, and $\left.T g(k d r l: R F P)^{l a 4}(34)\right]$ transgenic lines were used in this study.

MSU crystal synthesis and injection into zebrafish larvae. Endotoxinfree MSU crystals were prepared from uric acid (Sigma-Aldrich) as previously described (59). For microinjection, MSU crystals (10 mg) were dissociated by repeated passage through 18 - and then 22-gauge 

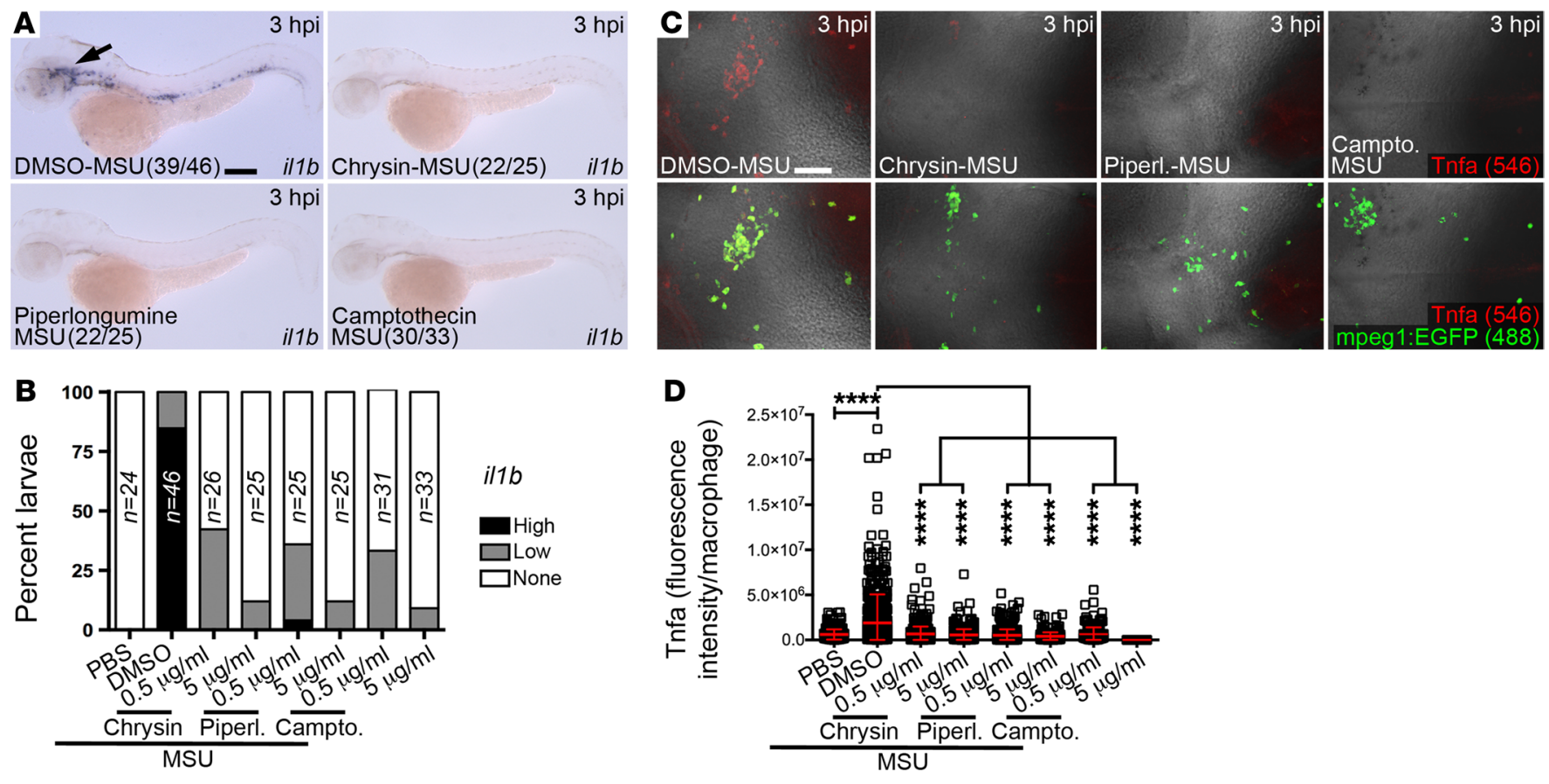

Figure 12. Drugs that inhibit irg1 expression suppress MSU crystal-driven macrophage activation. (A) Expression of il1b in MSU crystal-injected DMSO-, chrysin-, piperlongumine-, and camptothecin-treated larvae. Black arrow marks illb expression in hindbrain. The numbers in parentheses represent the frequency of larvae with the indicated phenotype. (B) Quantification of illb expression, as detected in A. (C) Immunofluorescence of Tnfa in the hindbrains of MSU crystal-injected DMSO-, chrysin-, piperlongumine-, and camptothecin-treated Tg(mpeg1:EGFP) larvae. The DMSO-MSU image is the same as in Figure 7C and Supplemental Figure 5G. (D) Quantification of Tnfa, as detected in C ( $n=15$ larvae/treatment). The DMSO-MSU sample is the same as in Figure 1C (3 hpi); Figure 3D; Figure 5F; Figure 7D; Figure 11B; Supplemental Figure 5, D and H; Supplemental Figure 7E; and Supplemental Figure 9E. Data were pooled from 2 independent experiments and represent the mean \pm SD. ${ }^{* * *} P<0.0001$, by 1-way ANOVA with Dunnett's post hoc test. Scale bars: 100 $\mu \mathrm{m}(\mathbf{A})$ and $50 \mu \mathrm{m}$ (C). Campto., camptothecin; Piperl., piperlongumine.

needles (Terumo) in filter-sterile PBS and sonicated, resulting in crystals of approximately $2 \mu \mathrm{m}$ in length. A 2 -nl volume of crystals was then delivered into the hindbrain ventricle of 2-dpf zebrafish larvae by microinjection. As controls, we used 2-nl volumes of filter-sterile PBS, uric acid (10 mM; Sigma-Aldrich; UO881), $1 \mu \mathrm{m}$ carboxylate-modified yellow-green fluorescent FluoSpheres (diluted 1:100 in filter-sterile PBS; Thermo Fisher Scientific; F8823), or calcium pyrophosphate crystals ( $5 \mathrm{mg} / \mathrm{ml}$ in filter-sterile PBS; InvivoGen).

Preparation of palmitic and stearic acids. Palmitic (C16:0; Sigma-Aldrich; PO500) and stearic (C18:0; Sigma-Aldrich; S4751) acids were conjugated with BSA as previously described (60), with a few minor modifications. Briefly, FAs were dissolved at $90^{\circ} \mathrm{C}$ in $100 \mathrm{mM}$ $\mathrm{NaOH}$ to a concentration of $50 \mathrm{mM}$, and then diluted $1: 5$ with a $5 \%$ solution of FA-free BSA (Roche; 10775835001$)$ at $37^{\circ} \mathrm{C}$. BSA-conjugated FAs were then filtered through a $0.2-\mu \mathrm{m}$ Acrodisc syringe filter and stored at $-20^{\circ} \mathrm{C}$.

Acute intravenous delivery of intralipid. Anesthetized 2-dpf $\mathrm{Tg}(\mathrm{kdrl}$ : RFP) or $T g$ (mpeg1:EGFP) larvae were injected into the sinus venosus with 4 separate 1 -nl boluses of intralipid (20\% emulsion; SigmaAldrich). To facilitate the live imaging of intralipid distribution within injected larvae, intralipid was supplemented with $50 \mu \mathrm{M}$ BODIPY FL $\mathrm{C}_{16}$ (InvitroGen; D3821).

Whole-mount ISH. Whole-mount ISH (WMISH) was performed using digoxigenin-labeled (DIG-labeled) and fluorescein-labeled (FLU-labeled) riboprobes (Roche) and BM Purple (Roche) color precipitation as previously described (61). Riboprobes to detect irg1 were generated as previously described (16), while those for $i l 1 b$ were generated from a cloned (705-bp) cDNA fragment using the following primer pair: forward, 5'-GCAAGAGGAACTTAACCAGCT-3' and reverse, 5'-TGCCGGTCTCCTTCCTGA-3'. For fluorescence WMISH, transcripts for $m m p 9$ and $k r t 4$ were labeled with antisense DIG- and FLU-labeled probes, respectively. Detection was performed using an Alexa Fluor 488 Tyramide Signal Amplification (TSA) Kit no. 2 (Invitrogen, Thermo Fisher Scientific) and an Alexa Fluor 555 TSA Kit no. 40 (Invitrogen, Thermo Fisher Scientific) according to the manufacturer's instructions.

Reverse transcription PCR. Reverse transcription PCR (RT-PCR) was used to determine MO specificity when SBMOs were used from 2-dpfSBMO-injected larvae. To assess Il1b SBMO, Tnfa SBMO, MyD88 SBMO, Irg1 SBMO1, and 2 specificities, the following primer pairs were used: forward, 5'-GCAAGAGGAACTTAACCAGCT-3', reverse, 5'-TGCCGGTCTCCTTCCTGA-3' (62); forward, 5'-GAGAGTTGCCTTTACCGCTG-3', reverse, 5'-CCGTAGGATTCAGAAAAGCG-3'; forward, 5'-CACGTTTCTTTTCGGGGTAACG-3', reverse, 5'-CTTCAGCACAGCAGATTAGGGC-3' (16); and forward, 5'-AAGCGGATGATTCTGGACAC-3', reverse, 5'-TACGGTCACTGGAGGSSSCC-3', respectively. Amplicons were cloned into the pGEM-T Easy Vector (Promega) for sequence verification of SBMO-induced modifications in splicing.

MO injection. MO (Gene Tools) were resuspended in sterile water and injected into $1 \times$ Danieau's solution. Efficacious doses for all MOs were determined empirically. RT-PCR was used to determine MO 

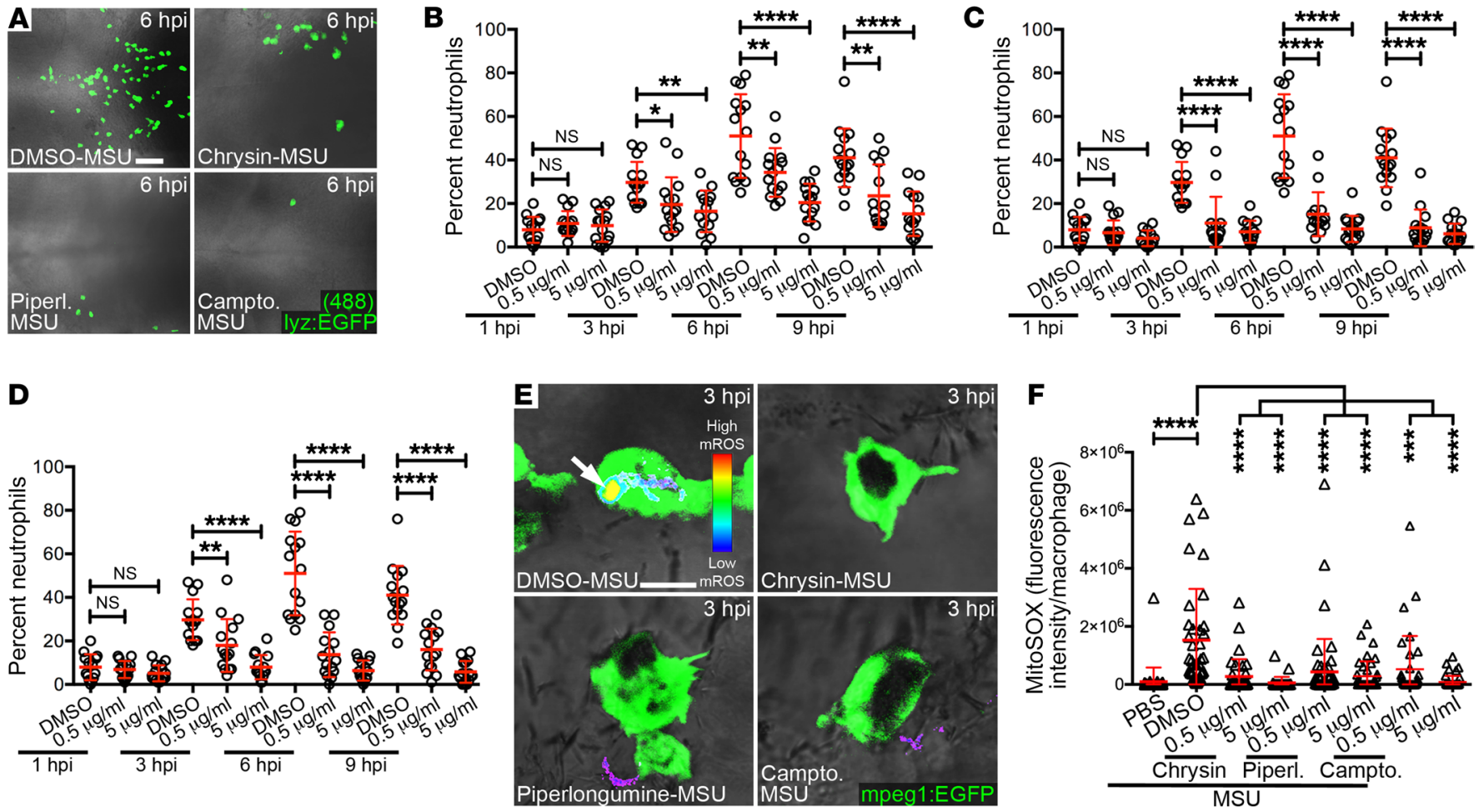

Figure 13. Drugs that inhibit irg1 expression suppress MSU crystal-driven neutrophil recruitment and macrophage-specific mROS production. (A) Immunofluorescence detection of neutrophils in MSU crystal-injected DMSO-, chrysin-, piperlongumine-, and camptothecin-treated Tg(lyz:EGFP) larvae. The DMSO-MSU image is the same as in Figure 2D and Supplemental Figure 5I. (B-D) Quantification of neutrophils, as detected in A, for chrysin (B), piperlongumine (C), and camptothecin (D) treatments ( $n=13-15$ larvae/treatment). The DMSO-MSU samples are the same as in Figure 2, B, E, and F; Figure 4, B and C; Figure 6C; Figure 8, B and C; Figure 11, D and E; Supplemental Figure 3D; Supplemental Figure 5, F and J; Supplemental Figure 7G; and Supplemental Figure 10, B and C. (E) Macrophage mROS production (white arrow) in the hindbrains of MSU crystal-injected DMSO-, chrysin-, piperlongumine-, and camptothecin-treated Tg(mpeg1:EGFP) larvae (MitoSOX signal is displayed as a heatmap, with warmer colors representing higher levels of mROS). (F) Quantification of macrophage-specific mROS production, as detected in $\mathbf{E}$ ( $n=10$ larvae/treatment). The DMSO-MSU sample is the same as in Figure 6F; Figure 8E; Supplemental Figure 7l; and Supplemental Figure 8, B and C. Data were pooled from 2 independent experiments and represent the mean \pm SD. ${ }^{*} P<0.05$, ${ }^{*} P<0.01,{ }^{* *} P<0.001$, and ${ }^{*}{ }^{*} P<0.0001$, by 1 -way ANOVA with Dunnett's post hoc test. Scale bars: $50 \mu \mathrm{m}(\mathbf{A})$ and $10 \mu \mathrm{m}(\mathbf{E})$.

specificity when SBMOs were used. The MO sequences (and doses) were as follows: control MO, 5'-CCTCTTACCTCAGTTACAATTTATA-3' (1.0 pmole); Illb SBMO, 5'-CCCACAAACTGCAAAATATCAGCTT-3' (62) (0.25 and 0.5 pmole); Tnfa SBMO, 5'-GCAGGATTTTCACCTTATGGAGCGT-3' (63) (0.25 and 0.5 pmole); Tnfa translation-blocking MO (ATGMO), 5'-AGCTTCATAATTGCTGTATGTCTTA-3' (64) (0.25 and 0.5 pmole); MyD88 SBMO, 5'-GGTTAAACACTGACCCTGTGGATC-3' (16) (0.25 and 0.5 pmole) (16); Irg1 SBMO1, 5'-TGCTGCCTCTAATTCATAAATGATC-3' (0.25 and 0.5 pmole) (16); and Irg1 SBMO2, 5'-TGAAAATCTGTTTTACCTTTTGGGA-3' (0.375 and 0.75 pmole).

Chemical treatments. The chemicals used in this study to pharmacologically inhibit specific signaling pathways and/or protein function were as follows: indomethacin (NSAID; Sigma-Aldrich); colchicine (microtubule destabilizer; Sigma-Aldrich); Z-VAD-FMK (pan-caspase inhibitor; InvivoGen); BAY11-7082 (inhibitor of IкB- $\alpha$ phosphorylation; InvivoGen); celastrol (NF- $\mathrm{KB}$ inhibitor; InvivoGen); triptolide (NF- $\mathrm{KB}$ inhibitor; InvivoGen); STAT3 IP (STAT3 inhibitor peptide PpYLKTK-mts; Calbiochem); AG490 (JAK2 inhibitor; InvivoGen); etomoxir (Cpt1 inhibitor; Sigma-Aldrich); MitoTEMPO (mROS inhibitor; Sigma-Aldrich); chrysin (Prestwick
Chemical); piperlongumine (Prestwick Chemical); and camptothecin (Prestwick Chemical). To assess the effects of drugs on illb or irg1 expression, Tnfa production, or neutrophil recruitment, drugs were diluted in E3 medium supplemented with 1\% DMSO and delivered by immersion. To assess the effects of the drugs on mROS production, a 1-nl volume of drug was microinjected into the hindbrain. Of note, no toxicity was observed for any of the chemical inhibitors used at the doses described.

Immunofluorescence. Immunofluorescence was carried out as previously described (16). The primary and secondary antibodies used for the detection of EGFP, Tnfa, and MyD88 and their dilutions were as follows: EGFP, chicken anti-GFP, 1:500 (Abcam; ab13970, lot GR236651-3) and goat anti-chicken Alexa Fluor 488, 1:500 (Invitrogen, Thermo Fisher Scientific; A11039/lot 1218174); Tnfa, rabbit anti-zebrafish Tnfa, 1:40 (AnaSpec; AS-55383, lot O10302) and goat anti-rabbit Alexa Fluor 546, 1:400 (Invitrogen, Thermo Fisher Scientific; A11010, lot 753750); MyD88, rabbit anti-zebrafish MyD88, 1:40 (AnaSpec; 55449, lot KK2103); and goat anti-rabbit Alexa Fluor 546, 1:400 (Invitrogen, Thermo Fisher Scientific; A11010, lot 753750).

Flow cytometry. Flow cytometry was performed as previously described (65), using a BD LSR II Flow Cytometer. 
A

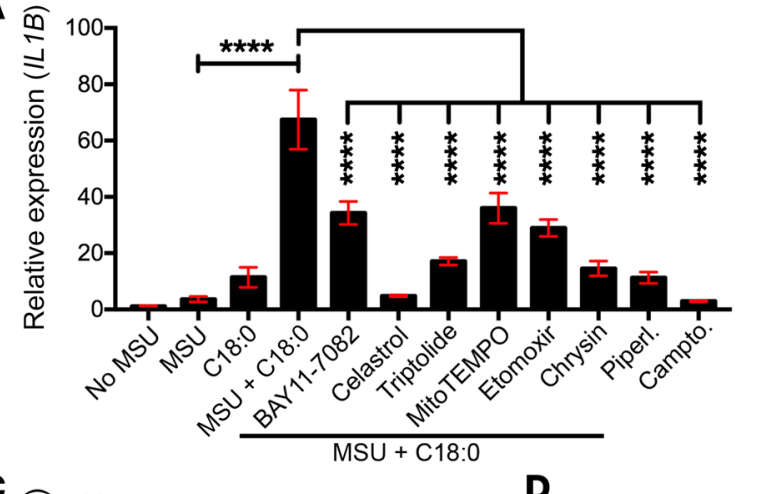

C

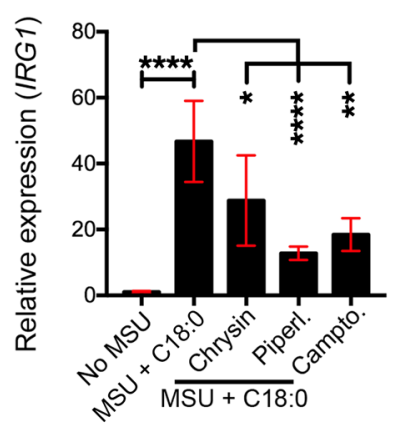

E

D

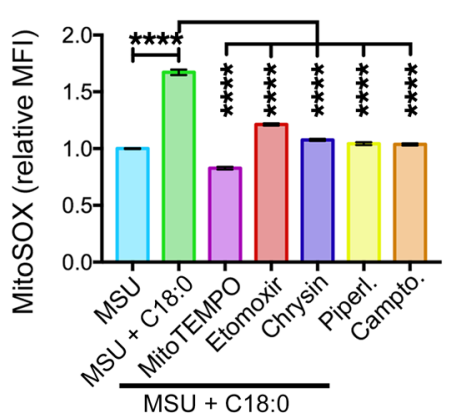

I
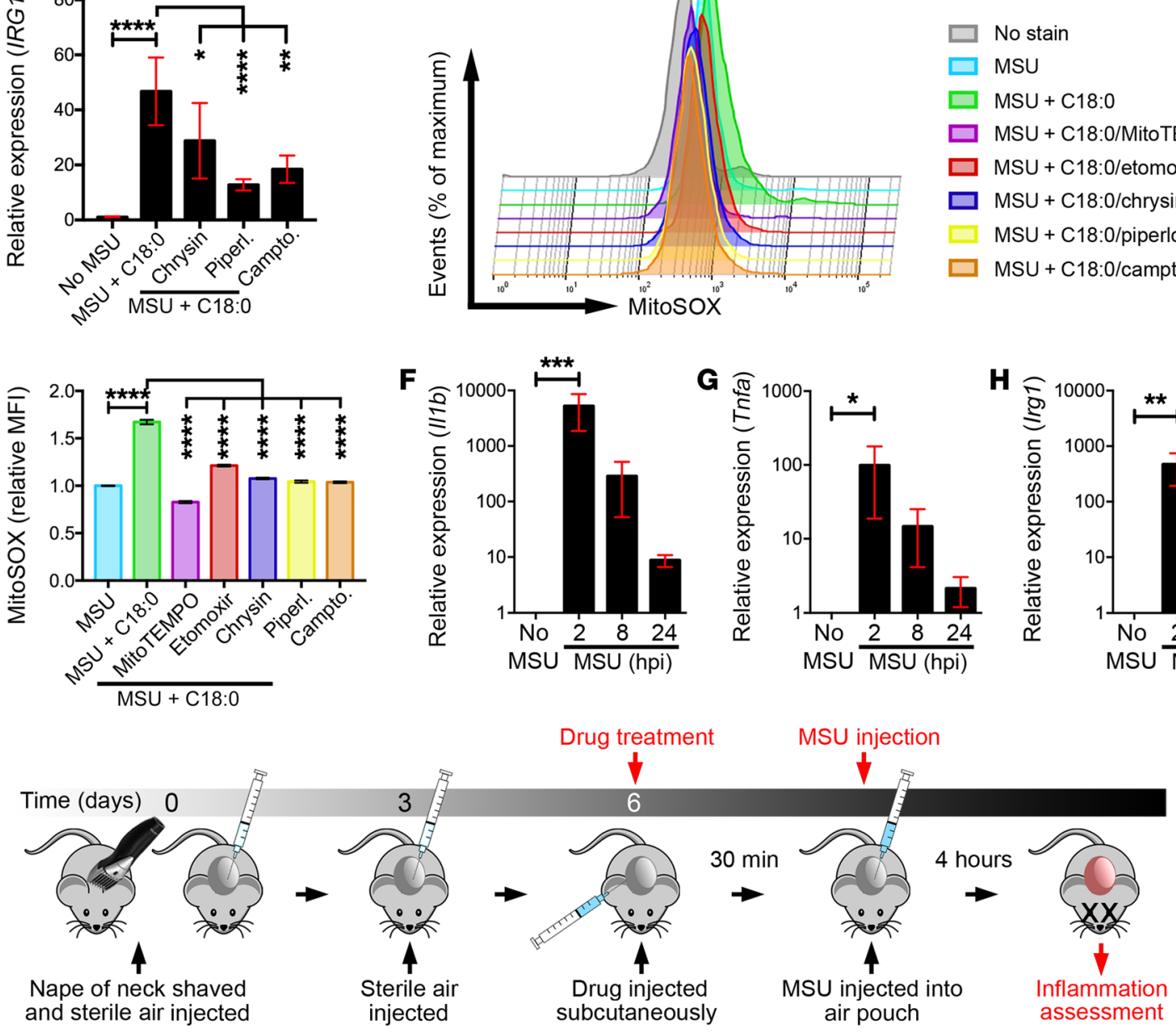

$\square$ No stain

$\square$ MSU

$\square \mathrm{MSU}+\mathrm{C} 18: 0$

$\square$ MSU + C18:0/MitoTEMPO

$\square$ MSU + C18:0/etomoxir

$\square \mathrm{MSU}+\mathrm{C18:0/ \text {chrysin }}$

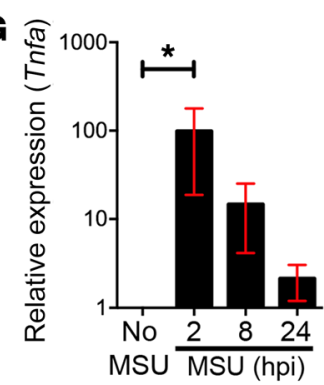

Sterile air

subcutaneously

air pouch

J

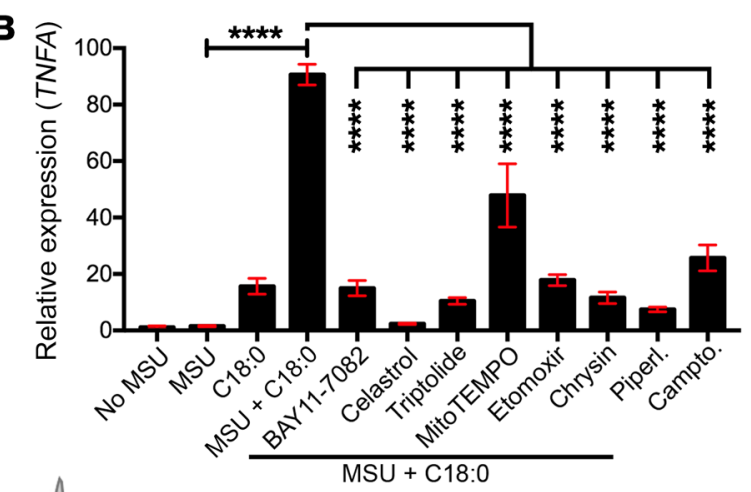

MSU + C18:0/piperlongumine

$\square$ MSU + C18:0/camptothecin

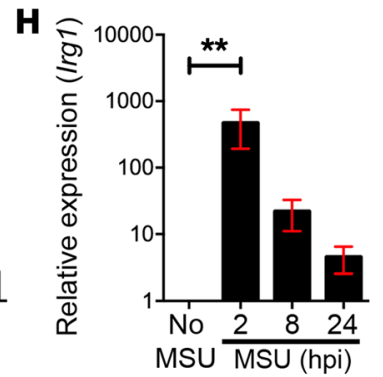

Chrysin

Piperlongumine

Camptothecin

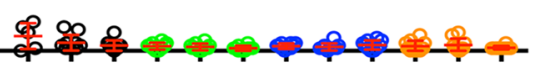

ญ

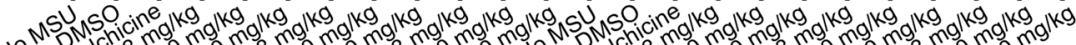


Figure 14. Stearic acid (C18:0) augments MSU crystal-stimulated expression of IL1B and TNFA expression in THP-1 cells through FAO-driven mROS production, and drugs that inhibit Irg1 expression suppress neutrophil recruitment in a murine air pouch model of acute gouty inflammation. (A and $\mathbf{B}$ ) Expression of IL1B (A) and TNFA (B) in THP-1 cells stimulated with MSU crystals, C18:0, MSU crystals plus [18:0, and MSU crystal plus C18:0 in the presence of BAY11-7082, celastrol, triptolide, MitoTEMPO, etomoxir, chrysin, piperlongumine, or camptothecin 6 hours after stimulation, relative to the no-MSU crystals control (qPCR, $n=5$ biological replicates). (C) Expression of IRG1 in THP-1 cells stimulated with MSU crystals plus C18:0 alone and in the presence of chrysin, piperlongumine, or camptothecin, measured after 6 hours of stimulation, relative to the no-MSU crystals control (qPCR, $n=5$ biological replicates). (D) Representative flow cytometric data showing MitoSOX fluorescence in THP-1 cells stimulated with MSU crystals, MSU crystals plus [18:0, and MSU crystals plus [18:0 in the presence of MitoTEMPO, etomoxir, chrysin, piperlongumine, or camptothecin, measured after 6 hours of stimulation (displayed as counts, percentage of maximum). (E) Quantification of MitoSOX signal (MFI), as detected in $\mathbf{D}$, relative to MSU crystal-treated cells $(n=5$ biological replicates). (F-H) Temporal expression of I/1b (F), Tnfa (G), and Irg1 (H) in peritoneal monocytes isolated from mice following intraperitoneal injection of MSU crystals, relative to the no-MSU crystals control (qPCR, $n=5$ mice/treatment). (I) Schematic of murine MSU crystal air pouch model of acute gouty inflammation and drug treatment strategy. (J) Effects of chrysin, piperlongumine, and camptothecin on neutrophil and monocyte numbers (measured as a percentage of leukocytes) in the murine MSU crystal air pouch model ( $n=5$ mice for the no-MSU crystals control; $n=10$ mice for all other treatments). Data represent the mean $\pm \mathrm{SD} .{ }^{*} P<0.05$, ${ }^{*} P<0.01$, ${ }^{* *} P<0.001$, and ${ }^{* * *} P<$ 0.0001 , by 1-way ANOVA with Dunnett's post hoc test (A-C, E, and J) and Kruskal-Wallis test $(\mathbf{F}-\mathbf{H})$.

Macrophage ablation experiments. For genetic depletion of macrophages, $\mathrm{Tg}$ (mpeg1:nfsB-mCherry) or $\mathrm{Tg}$ (mpeg1:nfsb-Cherry;lyz:EGFP) double-transgenic larvae (for neutrophil recruitment experiments) were treated with either 1 or $5 \mathrm{mM}$ metronidazole at 30 hpf for 18 hours. For liposome clodronate-mediated ablation, liposomes (as supplied by ClodronateLiposomes.org) were injected as previously described (66). Of note, given the requirement of blood circulation for normal, definitive hematopoiesis (67), only liposome-injected larvae with unaffected blood flow were used for subsequent experiments.

CRISPR-Cas9. CRISPR gRNA targets were selected and gRNAs were designed using the CRISPRdirect Web server (https://crispr. dbcls.jp/) (68). The primer pairs used to construct Irg1 gRNA no. 1 (to target exon 2) and Irg1 gRNA no. 2 (to target exon 5) were as follows: forward, 5'-TAGGTGTTAAAGACCGGTGTTC-3', reverse, 5'-AAACGAACACCGGTCTTTAACA-3'; and forward, 5'-TAGGTGGCAGCGTTGGCCATGG-3', reverse, 5'-AAACCCATGGCCAACGCTGCCA-3', respectively. The primer pairs were annealed and ligated into the T7cas9sgRNA2 vector (Addgene; 46759) that was linearized with BsmBI, BglII, and SalI (New England Biolabs), using T4 DNA ligase (New England Biolabs). Following transformation, single colonies were selected for sequencing confirmation. Sequence-confirmed vectors were then linearized with BamHI (New England Biolabs), and gRNA transcripts were generated using the MEGAshortscript T7 Kit (Invitrogen, Thermo Fisher Scientific). Cas 9 mRNA was in vitro transcribed from a Not I-linearized pCS2-nls-cCas9-nls plasmid (Addgene; 47929) using the mMESSAGE mMACHINE SP6 Kit (Invitrogen, Thermo Fisher Sci- entific). Irg1 gRNAs (75 pg) and Cas 9 mRNA (150 pg) were injected into single-cell-stage embryos. To validate CRISPR-Cas9-mediated genome editing, individual 2-dpf gRNA/Cas9-injected larvae were lysed in $10 \mu \mathrm{l}$ alkaline lysis buffer $(25 \mathrm{mM} \mathrm{NaOH}, 0.2 \mathrm{mM}$ EDTA) before addition of $1 \mu \mathrm{l}$ neutralization buffer ( $40 \mathrm{mM}$ Tris, $\mathrm{pH}$ 8.0) to isolate genomic DNA. Amplicons were then generated using primers immediately upstream and downstream of the gRNA targets in exons 2 and 5, respectively, (exon 2, forward, 5'-TTACGGACAGTTTTGGGGCA-3'; exon 5, reverse, 5'-TAGAAGGACCCGAAGCCAGA-3'). The PCR products were subsequently cloned into pCR-Blunt II-TOPO (Thermo Fisher Scientific) for sequencing and confirmation of genome editing.

Genetic inhibition of $N F-\kappa B$ signaling. To specifically inhibit NF- $\kappa \mathrm{B}$ signaling within macrophages, a macrophage-restricted dominant-negative ikbaa (dnikbaa) GFP fusion overexpression construct was generated by subcloning the p5E-mpeg1 (gift of Stephen Renshaw, University of Sheffield, Sheffield, United Kingdom), pME-dnikbaa (28), and p3E-V2AEGFP-pA (28) (gifts of Feng Liu, Institute of Zoology, Chinese Academy of Sciences, Beijing, China) entry vectors into the pDestTol2CG destination vector [containing the cardiac myosin light chain 2(cmlc2):EGFP transgenesis marker] from the Tol2kit (69). The resulting Tol2 mpeg1:dnikbaa-GFP construct (25 pg) was coinjected with in vitro-transcribed transposase mRNA (25 pg) at the single-cell stage.

Live imaging intracellular $\mathrm{H}_{2} \mathrm{O}_{2}$ with $\mathrm{HyPer}$ constructs. To express HyPer ubiquitously throughout larvae, single-cell-stage embryos were injected with $250 \mathrm{pg}$ in vitro-transcribed HyPer mRNA using the mMESSAGE mMACHINE SP6 Kit (Invitrogen, Thermo Fisher Scientific) and pCS2-HyPer as a template, as previously described (70). To restrict HyPer expression to macrophages, we generated a Tol2 UAS:HyPer construct using a pME-HyPer construct (gift of Stephen Renshaw, University of Sheffield, Sheffield, United Kingdom) and the p5E-UAS and pDestTol2CG Gateway constructs from the Tol2kit (69). Coinjection of this construct (25 pg) with a Tol2 mpeg1:Gal4FF construct $(25 \mathrm{pg})$ and in vitro-transcribed transposase mRNA ( $25 \mathrm{pg}$ ) into single-cell-stage embryos resulted in macrophage-restricted HyPer expression. HyPer fluorescence emission was detected at wavelengths of 505 to 510 and 510 to 525 $\mathrm{nm}$ following 405-nm and 488-nm excitation using an Olympus FluoView FV1000 scanning confocal microscope, as previously described (71). Raw images were processed and HyPer ratios generated as previously described (37). A HyPer ratio image (HyPer ${ }_{488} /$ $\mathrm{HyPer}_{405}$ ) was then generated and displayed as a heatmap, with warmer colors representing higher ratios being indicative of elevated intracellular $\mathrm{H}_{2} \mathrm{O}_{2}$ levels. To measure HyPer ratios before and after $\mathrm{H}_{2} \mathrm{O}_{2}$ microinjection, larvae were anesthetized and mounted in $1 \%$ low-melting-point agarose. Single scans were then performed through individual HyPer-expressing macrophages before a 1-nl volume of either filter-sterile PBS or $50 \mathrm{\mu M} \mathrm{H}_{2} \mathrm{O}_{2}$ was injected. Following injection, the same macrophages were imaged while maintaining identical imaging parameters to ensure that any differences were not an artifact of altered image acquisition settings. For each HyPer-expressing macrophage, a peak $\mathrm{HyPer}_{488} / \mathrm{HyPer}_{405}$ ratio was quantified by positioning the line-intensity measurement tool through the macrophage. HyPer ${ }_{488} / \mathrm{HyPer}_{405}$ ratio changes for individual HyPer-expressing macrophages were then normalized (with "before" HyPer ${ }_{488} / \mathrm{HyPer}_{405}$ ratios =1). 
Live imaging macrophage-specific mROS. To live image and quantify macrophage-specific mROS production, $50 \mu \mathrm{M}$ MitoSOX Red Mitochondrial Superoxide Indicator (Invitrogen, Thermo Fisher Scientific; M36008) was injected into the hindbrain ventricle of 2-dpf $\mathrm{Tg}$ (mpeg1:EGFP) larvae, as previously described (16).

Confocal imaging. Live imaging of MitoSOX and HyPer and timelapse imaging were performed on an Olympus FV1000 FluoView laser-scanning confocal microscope equipped with an incubation chamber. All other confocal imaging was performed using a Nikon C1 Eclipse confocal microscope. Quantification from confocal imaging was performed using Volocity Image Analysis Software (version 6.3, PerkinElmer). When confocal imaging was used to detect differences between treatment and control samples, identical imaging parameters were used to ensure that any differences were not an artifact of altered image acquisition settings. When quantifying neutrophils and macrophages within the hindbrain region, the following parameters were used: $512 \times 512$ pixels and $40 \times 3 \mu \mathrm{m} \mathrm{Z}$-stacks (extending from the dorsal-most surface of the hindbrain). When quantifying Tnfa and MyD88 within individual macrophages, the following parameters were used: $512 \times 512$ pixels and $40 \times 2 \mu \mathrm{m} Z$-stacks (extending from the dorsal-most surface of the hindbrain). Care was taken to image larvae at the exact same anatomical location. When live imaging drug-treated $\mathrm{Tg}$ (irg1:EGFP;mpeg1:nfsB-mCherry) larvae, larvae were anesthetized following MSU crystal injection and mounted in 1\% lowmelting-point agarose in E3 media supplemented with $125 \mu \mathrm{g} / \mathrm{ml}$ tricaine and $0.25 \mu \mathrm{g} / \mathrm{ml}$ chrysin, piperlongumine, or camptothecin (the highest drug dose tolerated during a 12-hour time-lapse experiment). The mean fluorescence intensity (MFI) of EGFP was then quantified within individual $\mathrm{mCherry}^{+}$macrophages in the hindbrain region using individual frames from time-lapse imaging experiments using Volocity Image Analysis Software.

Drug repositioning screen to identify inhibitors of irg1 expression. We screened the Prestwick Chemical Library (Prestwick Chemical) to identify drugs that inhibit irg1 expression in macrophages. The Prestwick Chemical Library contains 1,280 mainly FDA-approved drugs selected for their pharmacological diversity and known bioavailability and safety in humans. Larvae ( $2 \mathrm{dpf}$ ) were transferred into screening medium (E3 medium supplemented with $1 \% \mathrm{DMSO}, 20 \mu \mathrm{M}$ metronidazole, $0.05 \mathrm{U} / \mathrm{ml}$ penicillin, $50 \mathrm{ng} / \mathrm{ml}$ streptomycin, and $1 \mathrm{mM}$ Tris, pH 7.4) (ref. 72) and arrayed into 48 -well tissue culture plates (7 larvae/well). Larvae were exposed (by immersion) to a 2-hour drug pretreatment at $5 \mu \mathrm{g} / \mathrm{ml}$ before injection of LPS $(0.8 \mathrm{mg} / \mathrm{ml})$ into the hindbrain ventricle while in drug. Larvae were then fixed 2 hours after LPS injection and assessed for irg1 expression by WMISH analysis using an Intavis InsituPro VSi ISH robot. Macrophage-restricted expression of irg1 was then compared with sterile PBS-injected and LPS-injected DMSO (no drug) controls.

Mouse peritonitis model of acute gouty inflammation. Urate peritonitis was induced in 8- to 11-week-old male C57Bl/6J mice (Malaghan Institute of Medical Research, Wellington, New Zealand), as previously described (73).

THP-1 cell culture. The human monocytic THP-1 cell line (a gift of John Fraser, University of Auckland, Auckland, New Zealand) was grown in RPMI 1640 Media (Sigma-Aldrich) supplemented with 10\% FBS (Invitrogen, Thermo Fisher Scientific) and $1 \mathrm{mM}$ sodium pyruvate (Sigma-Aldrich) at $37^{\circ} \mathrm{C}$ in $5 \% \mathrm{CO}_{2}$. Cells were seeded at 1.5 $\times 10^{6}$ cells per well in 24-well plates and stimulated with $5 \mu \mathrm{M}$ PMA for 3 hours. Cells were washed twice in culture media and incubated overnight. Cells were washed again, transferred into culture media supplemented with $1 \%$ FA-free BSA, and treated with $500 \mu \mathrm{g} / \mathrm{ml} \mathrm{MSU}$ crystals or $200 \mu \mathrm{M}$ stearic acid (C18:0), alone and combined, or were left untreated (no MSU control) for 6 hours. For drug treatments, the following drugs were included: BAY11-7082 $(20 \mu \mathrm{M})$, celastrol $(20$ $\mu \mathrm{M})$, triptolide $(100 \mathrm{nM})$, MitoTEMPO $(250 \mu \mathrm{M})$, etomoxir $(250 \mu \mathrm{M})$, chrysin $(10 \mu \mathrm{M})$, piperlongumine $(10 \mu \mathrm{M})$, and camptothecin $(10 \mu \mathrm{M})$. Cells were then washed twice in PBS, and RNA was extracted for quantitative PCR (qPCR) analysis.

$R N A$ isolation and $q P C R$. Total RNA was isolated from larval zebrafish, mouse peritoneal monocytes/macrophages, and THP-1 cells using TRIzol Reagent (Life Technologies, Thermo Fisher Scientific), and cDNA was synthesized using SuperScript III Reverse Transcriptase (Life Technologies, Thermo Fisher Scientific). For larval zebrafish and THP-1 samples, qPCR was performed in technical quadruplicate using a QuantStudio 6K Flex Real-Time PCR System (Life Technologies, Thermo Fisher Scientific) for zebrafish cxcl8-l1: forward, 5'-GTCGCTGCATTGAAACAGAA-3', reverse, 5'-CTTAACCCATGGAGCAGAGG-3' (74) and cxcl8-l2: forward, 5'-GCTGGATCACACTGCAGAAA-3', reverse, 5'-TGCTGCAAACTTTTCCTTGA-3') (ref. 74); and human IRG1: forward, 5'-GTTAACTACACTTCTCAAAGGACCC-3', reverse, 5'-TACAAACCAAATCATTGAATCTCCC-3' (ref. 75); IL1B: forward, 5'-TGGAAGGAGCACTTCATCTG-3', reverse, 5'-TCAGCCAATCTTCATTGCTC-3'; and TNFA: forward, 5'-TGGAAGGAGCACTTCATCTG-3', reverse, 5'-TGAGGTACAGGCCCTCTGAT-3' using PerfeCTa SYBR Green FastMix (Quantabio). Expression levels were normalized to efl $\alpha$ : forward, 5'-TGCCTTCGTCCCAATTTCAG-3', reverse, 5'-TACCCTCCTTGCGCTCAATC-3' and GAPDH: forward, 5'-TGCACCACCAACTGCTTAGC-3', reverse, 5'-GGCATGGACTGTGGTCATGAG-3' for zebrafish and human samples, respectively. For mouse samples, qPCR was performed in technical duplicate using a QuantStudio 12K Flex RealTime PCR System (Life Technologies, Thermo Fisher Scientific) for murine Il1b (Mm00434228_m1), Tnfa (Mm00443258_m1), and Irg1 (Mm01224532_m1), and expression levels were normalized to 18s rRNA (Mm03928990_g1). For all samples, expression levels were calculated using the $\Delta \Delta \mathrm{Ct}$ method.

Flow cytometric analysis of $m R O S$ production. mROS were detected within THP-1 cells as previously described (76). In brief, following treatments, cells were incubated with $2.5 \mu \mathrm{M}$ MitoSOX (Invitrogen, Thermo Fisher Scientific; M36008) for 30 minutes, washed in PBS, removed from plates with ice-cold PBS supplemented with $1 \mathrm{mM}$ EDTA, pelleted, and resuspended in ice-cold PBS supplemented with $1 \%$ FBS. Fluorescence intensities were then measured using a BD LSR II Flow Cytometer.

Murine air pouch model of acute gouty inflammation. The in vivo testing of chrysin, piperlongumine, and camptothecin was performed under contract with Washington Biotech Inc., using a murine air pouch model of acute gouty inflammation. In brief, 5to 6-week-old male BALB/c mice (Envigo) were anesthetized and the nape of the neck shaved. On days 0 and 3, sterile air $(6 \mathrm{ml})$ was injected subcutaneously to generate an air pouch that morphologically resembled the joint synovium. Mice were randomly divided into the following groups: $n=5$ mice for no MSU control group and $n=10$ mice each for no drug group (DMSO), positive control group (1 mg/kg colchicine), and chrysin, piperlongumine, and camptoth- 
ecin treatment groups. Freshly prepared MSU crystals (30 mg in 3 $\mathrm{ml}$ ) were injected into the air pouch 30 minutes after subcutaneous delivery of drugs. Four hours after MSU crystal injection, exudates were collected from the air pouches, and total white cell and differential leukocyte counts were performed using an Abbott CELLDYN Hematology Analyzer.

Statistics. All data are presented as the mean \pm SD. Statistical significance of differences between 2 groups was determined using an unpaired, 2-tailed $t$ test. For multiple comparisons among control and experimental groups, a 1-way ANOVA with Dunnett's post hoc correction was used. $P$ values of less than 0.05 were considered statistically significant. All statistical analyses were performed using GraphPad Prism 5.0 (GraphPad Software). For the assessment of treatment effects on neutrophil recruitment to MSU crystals in zebrafish, larvae were collected from 2 independent experiments and then pooled, and neutrophils from 13 to 15 larvae were quantified. Comparisons were made of control groups specific to the experimental technique. No significant differences were observed within each time point between any of the control groups used (Supplemental Figure 13A), supporting the reproducibility of our control data. To determine the treatment effects on Tnfa production, larvae were collected in a similar fashion, and Tnfa levels were quantified in macrophages from 15 larvae. For mROS production, measurements were taken from approximately 10 larvae from at least 2 independent experiments. A similar comparison between the control groups used for Tnfa and mROS production also revealed no significant differences (Supplemental Figure 13, B and C).

Study approval. All zebrafish research was conducted with the approval of the University of Auckland Animal Ethics Committee. For mouse studies, the peritonitis model experiments were performed in accordance with procedures approved by the Victoria University Animal Ethics Committee, while the air pouch model study was carried out following ethical standards for care and use of laboratory animals under Public Health Services (Baltimore, USA)/NIH regulations and the study approved by the IACUC at Washington Biotechnology, Inc.

\section{Author contributions}

$\mathrm{CJH}$ devised and performed experiments and wrote the manuscript. LES performed the drug-repositioning screen. LML, EA, and MVDL helped perform experiments. BP and JLH generated MSU crystals and performed expression analysis of mouse monocytes/macrophages. DB helped with cell culture experiments. GJL provided the mpeg1 constructs and lines. JWA, ND, KEC, and PSC helped devise experiments and write the manuscript.

\section{Acknowledgments}

This work was supported by grants awarded by the Marsden Fund, the Royal Society of New Zealand, and the Health Research Council of New Zealand (to CJH) and by the Ministry of Business, Innovation and Employment of New Zealand (to PSC). GJL was supported by the National Health and Medical Research Council (grants 637394, 1044754, 1069284, and 1086020). The Australian Regenerative Medicine Institute is supported by funds from the State Government of Victoria and the Australian Federal Government. We thank the Biomedical Imaging Research Unit at the School of Medical Sciences of the University of Auckland for microscopy assistance.

Address correspondence to: Christopher J. Hall, Department of Molecular Medicine and Pathology, School of Medical Sciences, University of Auckland, Private Bag 92019, Auckland, New Zealand. Phone: 64.9.923.2910; Email: c.hall@auckland.ac.nz.

LES's present address is: Universite Libre de Bruxelles, Brussels, Belgium.

MvdK's present address is: UMC Utrecht, Utrecht, Netherlands.
1. Zhu Y, Pandya BJ, Choi HK. Prevalence of gout and hyperuricemia in the US general population: the National Health and Nutrition Examination Survey 2007-2008. Arthritis Rheum. 2011;63(10):3136-3141.

2. Faires JS, McCarty DJ. Acute arthritis in man and dog after intrasynovial injection of sodium urate crystals. Lancet. 1962;280:682-685.

3. Lindsay K, Gow P, Vanderpyl J, Logo P, Dalbeth $\mathrm{N}$. The experience and impact of living with gout: a study of men with chronic gout using a qualitative grounded theory approach. JClin Rheumatol. 2011;17(1):1-6.

4. Dalbeth N, Merriman TR, Stamp LK. Gout. Lancet. 2016;388(10055):2039-2052.

5. Kuncl RW, Duncan G, Watson D, Alderson K, Rogawski MA, Peper M. Colchicine myopathy and neuropathy. N Engl J Med. 1987;316(25):1562-1568.

6. Zhu Y, Pandya BJ, Choi HK. Comorbidities of gout and hyperuricemia in the US general population: NHANES 2007-2008. Am J Med. 2012;125(7):679-687.e1.

7. Schumacher HR Jr, et al. Randomised double blind trial of etoricoxib and indometacin in treatment of acute gouty arthritis. BMJ. 2002;324(7352):1488-1492.
8. So AK, Martinon F. Inflammation in gout: mechanisms and therapeutic targets. Nat Rev Rheumatol. 2017;13(11):639-647.

9. Martin WJ, Harper JL. Innate inflammation and resolution in acute gout. Immunol Cell Biol. 2010;88(1):15-19.

10. Martinon F, Pétrilli V, Mayor A, Tardivel A, Tschopp J. Gout-associated uric acid crystals activate the NALP3 inflammasome. Nature. 2006;440(7081):237-241.

11. Mielants H, Veys EM, de Weerdt A. Gout and its relation to lipid metabolism. I. Serum uric acid, lipid, and lipoprotein levels in gout. Ann Rheum Dis. 1973;32(6):501-505.

12. Mielants H, Veys EM, de Weerdt A. Gout and its relation to lipid metabolism. II. Correlations between uric acid, lipid, and lipoprotein levels in gout. Ann Rheum Dis. 1973;32(6):506-509.

13. Joosten LA, et al. Engagement of fatty acids with Toll-like receptor 2 drives interleukin-1 $\beta$ production via the ASC/caspase 1 pathway in monosodium urate monohydrate crystal-induced gouty arthritis. Arthritis Rheum. 2010;62(11):3237-3248.

14. O’Neill LA, Pearce EJ. Immunometabolism governs dendritic cell and macrophage function. J Exp Med. 2016;213(1):15-23.
15. West AP, Shadel GS, Ghosh S. Mitochondria in innate immune responses. Nat Rev Immunol. 2011;11(6):389-402.

16. Hall CJ, et al. Immunoresponsive gene 1 augments bactericidal activity of macrophage-lineage cells by regulating $\beta$-oxidation-dependent mitochondrial ROS production. Cell Metab. 2013;18(2):265-278.

17. Michelucci A, et al. Immune-responsive gene 1 protein links metabolism to immunity by catalyzing itaconic acid production. Proc Natl Acad Sci US A. 2013;110(19):7820-7825.

18. Pessler F, et al. Identification of novel monosodium urate crystal regulated mRNAs by transcript profiling of dissected murine air pouch membranes. Arthritis Res Ther. 2008;10(3):R64.

19. Hall CJ, et al. Epidermal cells help coordinate leukocyte migration during inflammation through fatty acid-fuelled matrix metalloproteinase production. Nat Commun. 2014;5:3880.

20. Ellett F, Pase L, Hayman JW, Andrianopoulos A, Lieschke GJ. mpeg1 promoter transgenes direct macrophage-lineage expression in zebrafish. Blood. 2011;117(4):e49-e56.

21. Busso N, Ea HK. The mechanisms of inflammation in gout and pseudogout (CPP-induced arthritis). Reumatismo. 2012;63(4):230-237. 
22. Khanna D, et al. 2012 American College of Rheumatology guidelines for management of gout. Part 2: therapy and antiinflammatory prophylaxis of acute gouty arthritis. Arthritis Care Res (Hoboken). 2012;64(10):1447-1461.

23. Martin WJ, Walton M, Harper J. Resident macrophages initiating and driving inflammation in a monosodium urate monohydrate crystal-induced murine peritoneal model of acute gout. Arthritis Rheum. 2009;60(1):281-289.

24. Okuda KS, et al. A zebrafish model of inflammatory lymphangiogenesis. Biol Open. 2015;4(10):1270-1280.

25. Bernut A, et al. Mycobacterium abscessus cording prevents phagocytosis and promotes abscess formation. Proc Natl Acad Sci US A. 2014;111(10):E943-E952.

26. Vojtech LN, Scharping N, Woodson JC, Hansen JD. Roles of inflammatory caspases during processing of zebrafish interleukin-1 $\beta$ in Francisella noatunensis infection. Infect Immun. 2012;80(8):2878-2885.

27. Liu-Bryan R, Scott P, Sydlaske A, Rose DM, Terkeltaub R. Innate immunity conferred by Toll-like receptors 2 and 4 and myeloid differentiation factor 88 expression is pivotal to monosodium urate monohydrate crystal-induced inflammation. Arthritis Rheum. 2005;52(9):2936-2946.

28. He Q, et al. Inflammatory signaling regulates hematopoietic stem and progenitor cell emergence in vertebrates. Blood. 2015;125(7):1098-1106.

29. Terkeltaub R, Zachariae C, Santoro D, Martin J, Peveri P, Matsushima K. Monocyte-derived neutrophil chemotactic factor/interleukin-8 is a potential mediator of crystal-induced inflammation. Arthritis Rheum. 1991;34(7):894-903.

30. Vickers AE. Characterization of hepatic mitochondrial injury induced by fatty acid oxidation inhibitors. Toxicol Pathol. 2009;37(1):78-88.

31. Cader MZ, et al. C13orf31 (FAMIN) is a central regulator of immunometabolic function. Nat Immunol. 2016;17(9):1046-1056.

32. Lai E, et al. Blood lipids affect rat islet blood flow regulation through $\beta$ (3)-adrenoceptors. Am JPhysiol Endocrinol Metab. 2014;307(8):E653-E663.

33. Carten JD, Bradford MK, Farber SA. Visualizing digestive organ morphology and function using differential fatty acid metabolism in live zebrafish. Dev Biol. 2011;360(2):276-285.

34. Huang H, Zhang B, Hartenstein PA, Chen JN, Lin S. NXT2 is required for embryonic heart development in zebrafish. BMC Dev Biol. 2005;5:7.

35. Yang Y, Bazhin AV, Werner J, Karakhanova S. Reactive oxygen species in the immune system. Int Rev Immunol. 2013;32(3):249-270.

36. Takada Y, Mukhopadhyay A, Kundu GC, Mahabeleshwar GH, Singh S, Aggarwal BB. Hydrogen peroxide activates NF- $\kappa \mathrm{B}$ through tyrosine phosphorylation of $\mathrm{I} \kappa \mathrm{B} \alpha$ and serine phosphorylation of $\mathrm{p} 65$ : evidence for the involvement of I $\mathrm{B} \alpha$ kinase and Syk protein-tyrosine kinase. J Biol Chem. 2003;278(26):24233-24241.

37. Niethammer P, Grabher C, Look AT, Mitchison TJ. A tissue-scale gradient of hydrogen peroxide mediates rapid wound detection in zebrafish. Nature. 2009;459(7249):996-999.

38. Zon LI, Peterson RT. In vivo drug discovery in the zebrafish. Nat Rev Drug Discov. 2005;4(1):35-44.

39. Sanderson LE, Chien AT, Astin JW, Crosier KE, Crosier PS, Hall CJ. An inducible transgene reports activation of macrophages in live zebrafish larvae. Dev Comp Immunol. 2015;53(1):63-69.

40. Edwards JC, Sedgwick AD, Willoughby DA. The formation of a structure with the features of synovial lining by subcutaneous injection of air: an in vivo tissue culture system. J Pathol. 1981;134(2):147-156.

41. Lee JY, Sohn KH, Rhee SH, Hwang D. Saturated fatty acids, but not unsaturated fatty acids, induce the expression of cyclooxygenase- 2 mediated through Toll-like receptor 4. J Biol Chem. 2001;276(20):16683-16689.

42. Lopes SM, Trimbo SL, Mascioli EA, Blackburn GL. Human plasma fatty acid variations and how they are related to dietary intake. Am JClin Nutr. 1991;53(3):628-637.

43. Pearce EL, Pearce EJ. Metabolic pathways in immune cell activation and quiescence. Immunity. 2013;38(4):633-643.

44. Mills EL, Kelly B, O’Neill LAJ. Mitochondria are the powerhouses of immunity. Nat Immunol. 2017;18(5):488-498.

45. Van den Bossche J, O'Neill LA, Menon D. Macrophage immunometabolism: where are we (going)? Trends Immunol. 2017;38(6):395-406.

46. Lampropoulou $\mathrm{V}$, et al. Itaconate links inhibition of succinate dehydrogenase with macrophage metabolic remodeling and regulation of inflammation. Cell Metab. 2016;24(1):158-166.

47. Ren K, et al. Suppression of IRG-1 reduces inflammatory cell infiltration and lung injury in respiratory syncytial virus infection by reducing production of reactive oxygen species. J Virol. 2016;90(16):7313-7322.

48. Ganta VC, Choi MH, Kutateladze A, Fox TE, Farber CR, Annex BH. A microRNA93IRF9-IRG1-itaconic acid pathway modulates M2-like-macrophage polarization to revascularize ischemic muscle. Circulation. 2017;135(24):2403-2425.

49. Papathanassiu AE, et al. BCAT1 controls metabolic reprogramming in activated human macrophages and is associated with inflammatory diseases. Nat Commun. 2017;8:16040.

50. Lirdprapamongkol K, et al. Chrysin overcomes TRAIL resistance of cancer cells through Mcl-1 downregulation by inhibiting STAT3 phosphorylation. Int JOncol. 2013;43(1):329-337.

51. Song B, Zhan H, Bian Q, Gu J. Piperlongumine inhibits gastric cancer cells via suppression of the JAK1,2/STAT3 signaling pathway. Mol Med Rep. 2016;13(5):4475-4480.

52. Cross-Knorr S, et al. RKIP phosphorylation and STAT3 activation is inhibited by oxaliplatin and camptothecin and are associated with poor prognosis in stage II colon cancer patients. BMC Cancer. 2013;13:463.

53. Venditto VJ, Simanek EE. Cancer therapies utilizing the camptothecins: a review of the in vivo literature. Mol Pharm. 2010;7(2):307-349.

54. Hsiang YH, et al. DNA topoisomerase I-mediated DNA cleavage and cytotoxicity of camptothecin analogues. Cancer Res. 1989;49(16):4385-4389.

55. Geeraerts X, Bolli E, Fendt SM, Van Ginderachter
JA. Macrophage metabolism as therapeutic target for cancer, atherosclerosis, and obesity. Front Immunol. 2017;8:289.

56. Feng $X$, et al. Chrysin attenuates inflammation by regulating M1/M2 status via activating PPAR $\gamma$. Biochem Pharmacol. 2014;89(4):503-514.

57. Davison JM, et al. Transactivation from Gal4VP16 transgenic insertions for tissue-specific cell labeling and ablation in zebrafish. Dev Biol. 2007;304(2):811-824.

58. Hall C, Flores MV, Storm T, Crosier K, Crosier P. The zebrafish lysozyme $\mathrm{C}$ promoter drives myeloid-specific expression in transgenic fish. BMC Dev Biol. 2007;7:42.

59. Dalbeth N, et al. Enhanced osteoclastogenesis in patients with tophaceous gout: urate crystals promote osteoclast development through interactions with stromal cells. Arthritis Rheum. 2008;58(6):1854-1865.

60. Cullberg KB, Larsen JØ, Pedersen SB, Richelsen B. Effects of LPS and dietary free fatty acids on MCP-1 in 3T3-L1 adipocytes and macrophages in vitro. Nutr Diabetes. 2014;4:e113.

61. Jowett T, Lettice L. Whole-mount in situ hybridizations on zebrafish embryos using a mixture of digoxigenin- and fluorescein-labelled probes. Trends Genet. 1994;10(3):73-74.

62. Nguyen-Chi M, Phan QT, Gonzalez C, Dubremetz JF, Levraud JP, Lutfalla G. Transient infection of the zebrafish notochord with E. coli induces chronic inflammation. Dis Model Mech. 2014;7(7):871-882.

63. López-Muñoz A, Sepulcre MP, Roca FJ, Figueras A, Meseguer J, Mulero V. Evolutionary conserved pro-inflammatory and antigen presentation functions of zebrafish IFN $\gamma$ revealed by transcriptomic and functional analysis. Mol Immunol. 2011;48(9-10):1073-1083.

64. Matthews RP, et al. TNF $\alpha$-dependent hepatic steatosis and liver degeneration caused by mutation of zebrafish S-adenosylhomocysteine hydrolase. Development. 2009;136(5):865-875.

65. Hall C, Flores MV, Chien A, Davidson A, Crosier $\mathrm{K}$, Crosier P. Transgenic zebrafish reporter lines reveal conserved Toll-like receptor signaling potential in embryonic myeloid leukocytes and adult immune cell lineages. J Leukoc Biol. 2009;85(5):751-765.

66. Astin JW, et al. Innate immune cells and bacterial infection in zebrafish. Methods Cell Biol. 2017;138:31-60.

67. North TE, et al. Hematopoietic stem cell development is dependent on blood flow. Cell. 2009;137(4):736-748.

68. Naito Y, Hino K, Bono H, Ui-Tei K. CRISPRdirect: software for designing CRISPR/Cas guide RNA with reduced off-target sites. Bioinformatics. 2015;31(7):1120-1123

69. Kwan KM, et al. The Tol2kit: a multisite gateway-based construction kit for Tol2 transposon transgenesis constructs. Dev Dyn. 2007;236(11):3088-3099.

70. Pase L, et al. Neutrophil-delivered myeloperoxidase dampens the hydrogen peroxide burst after tissue wounding in zebrafish. Curr Biol. 2012;22(19):1818-1824.

71. Yoo SK, Starnes TW, Deng Q, Huttenlocher A. Lyn is a redox sensor that mediates 
leukocyte wound attraction in vivo. Nature. 2011;480(7375):109-112.

72. Murphey RD, Stern HM, Straub CT, Zon LI. A chemical genetic screen for cell cycle inhibitors in zebrafish embryos. Chem Biol Drug Des. 2006;68(4):213-219.

73. Dalbeth N, et al. Role of miR-146a in regulation of the acute inflammatory response to monosodium urate crystals. Ann Rheum Dis. 2015;74(4):786-790.

74. de Oliveira S, Lopez-Muñoz A, Martínez-Navarro FJ, Galindo-Villegas J, Mulero V, Calado Â. Cxcl8-11 and Cxcl8-12 are required in the zebrafish defense against Salmonella Typhimurium. Dev Comp Immunol. 2015;49(1):44-48.

75. Liu X, Wu XP, Zhu XL, Li T, Liu Y. IRG1 increases
MHC class I level in macrophages through STATTAP1 axis depending $\triangle 1$ on NADPH oxidase mediated reactive oxygen species. Int Immunopharmacol. 2017;48:76-83.

76. Lee S, Wi SM, Min Y, Lee KY. Peroxiredoxin-3 is involved in bactericidal activity through the regulation of mitochondrial reactive oxygen species. Immune Netw. 2016;16(6):373-380. 